\title{
HOXA9 regulates BRCA1 expression to modulate human breast tumor phenotype
}

\author{
Penney M. Gilbert, ${ }^{1,2}$ Janna K. Mouw, ${ }^{3}$ Meredith A. Unger, ${ }^{4}$ Johnathon N. Lakins, ${ }^{3}$ \\ Mawuse K. Gbegnon, ${ }^{1,2}$ Virginia B. Clemmer, ${ }^{5}$ Miriam Benezra, ${ }^{6}$ Jonathan D. Licht, ${ }^{7}$ \\ Nancy J. Boudreau, ${ }^{3}$ Kelvin K.C. Tsai, ${ }^{8}$ Alana L. Welm, ${ }^{9}$ Michael D. Feldman, ${ }^{2}$ \\ Barbara L. Weber, ${ }^{4}$ and Valerie M. Weaver ${ }^{1,2,3,10}$
}

\begin{abstract}
${ }^{1}$ Institute for Medicine and Engineering, and ${ }^{2}$ Department of Pathology, University of Pennsylvania, Philadelphia, Pennsylvania, USA. ${ }^{3}$ Center for Bioengineering and Tissue Regeneration and Department of Surgery, UCSF, San Francisco, California, USA. ${ }^{4}$ Abramson Family Cancer Research Institute, University of Pennsylvania, Philadelphia, Pennsylvania, USA. ${ }^{5}$ St. Francis Hospital, Wilmington, Delaware, USA. ${ }^{6}$ Mount Sinai School of Medicine, New York, New York, USA. ${ }^{7}$ Department of Medicine, Northwestern University Feinberg School of Medicine, Chicago, Illinois, USA. ${ }^{8}$ Graduate Institute of Clinical Research, Taipei Medical University, Taipei, Taiwan. ${ }^{D}$ Department of Oncological Sciences, Huntsman Cancer Institute, University of Utah, Salt Lake City, Utah, USA. ${ }^{10}$ Department of Anatomy and Department of Bioengineering and Therapeutic Sciences, Eli and Edythe Broad Center of Regeneration Medicine and Stem Cell Research, and UCSF Helen Diller Comprehensive Cancer Center, UCSF, San Francisco, California, USA.
\end{abstract}

\begin{abstract}
Breast cancer 1, early onset (BRCA1) expression is often reduced in sporadic breast tumors, even in the absence of BRCA1 genetic modifications, but the molecular basis for this is unknown. In this study, we identified homeobox A9 (HOXA9) as a gene frequently downregulated in human breast cancers and tumor cell lines and noted that reduced HOXA9 transcript levels associated with tumor aggression, metastasis, and patient mortality. Experiments revealed that loss of HOXA9 promoted mammary epithelial cell growth and survival and perturbed tissue morphogenesis. Restoring HOXA9 expression repressed growth and survival and inhibited the malignant phenotype of breast cancer cells in culture and in a xenograft mouse model. Molecular studies showed that HOXA9 restricted breast tumor behavior by directly modulating the expression of BRCA1. Indeed, ectopic expression of wild-type BRCA1 phenocopied the tumor suppressor function of HOXA9, and reducing BRCA1 levels or function inhibited the antitumor activity of HOXA9. Consistently, HOXA9 expression correlated with BRCA1 in clinical specimens and with tumor aggression in patients lacking estrogen receptor/progesterone receptor expression in their breast tissue. These findings indicate that HOXA9 restricts breast tumor aggression by modulating expression of the tumor suppressor gene BRCA1, which we believe provides an explanation for the loss of BRCA1 expression in sporadic breast tumors in the absence of $B R C A 1$ genetic modifications.
\end{abstract}

\section{Introduction}

Developmental regulators that specify tissue morphogenesis are often functionally subverted in adult tissues to promote cancer $(1,2)$. In particular, the homeobox (HOX) gene family of morphogenic regulators is critical for the establishment of embryonic patterning during embryogenesis and for the maintenance of tissue differentiation in the adult organism (3). HOX genes are transcription factors that regulate the expression of multiple genes that influence cell growth and viability and that mediate stromal-epithelial interactions to drive tissue-specific differentiation (2). HOX expression is frequently perturbed in tumors (3), in which they can act as oncogenes by promoting cell growth and invasion $(4,5)$ or as tumor suppressors (TSs), because they can alter cell survival and morphogenesis (6-9). HOX genes are especially important in the mammary gland, which undergoes repeated rounds of developmental cycles in the adult organism $(10,11)$. In the breast, HOX genes have been implicated in the control of embryonic development, branching morphogenesis, and hormonally controlled differentiation $(10,12)$, and HOX genes are frequently lost or overexpressed in breast tumors (12). Nevertheless, the molecular mechanisms whereby HOX genes regulate mammary development and might modulate breast cancer remain poorly defined.

Authorship note: Penney M. Gilbert and Janna K. Mouw contributed equally to this work.

Conflict of interest: The authors have declared that no conflict of interest exists. Citation for this article: J Clin Invest. 2010;120(5):1535-1550. doi:10.1172/JCI39534.
Women with hereditary mutations in BRCA1 are predisposed to develop breast and ovarian cancers (13). BRCA1 maintains genome integrity through its ubiquitin ligase activity (14) and regulates transcription to modulate the cellular stress response $(15,16)$. BRCA1 has an established role as a regulator of mammary epithelial cell (MEC) growth, survival, morphogenesis, and transformation (17-20). Moreover, loss of BRCA1 expression and/or function is associated with increased breast tumor aggression, enhanced cancer metastasis, and a poor clinical prognosis (21). There is also a clinical association between familial BRCA1 tumors and an aggressive, triple-negative, basal-like breast cancer phenotype (22). Interestingly, many sporadic breast cancers show decreased BRCA1 expression and display a "BRCA1-like" phenotype, despite the absence of genetic deletions, methylation, or haploinsufficiency $(23,24)$. The molecular mechanisms leading to reduced BRCA1 expression and/or function in this group of aggressive, sporadic breast cancers remain unclear $(20,25)$. Studies designed to elucidate molecular modifiers of BRCA1 have identified negative regulators, which in some cases are overexpressed during breast tumorigenesis $(26-29,30,31)$. Conversely, the identification of transcription factors that positively and directly regulate BRCA1 expression and whose expression might be concurrently lost during malignant transformation has proven elusive.

BRCA1 expression and MEC proliferation are functionally linked, suggesting BRCA1 could regulate mammary gland development and homeostasis and inhibit tumorigenesis by restricting MEC growth (32-34). BRCA1 also modulates mammary gland 
A
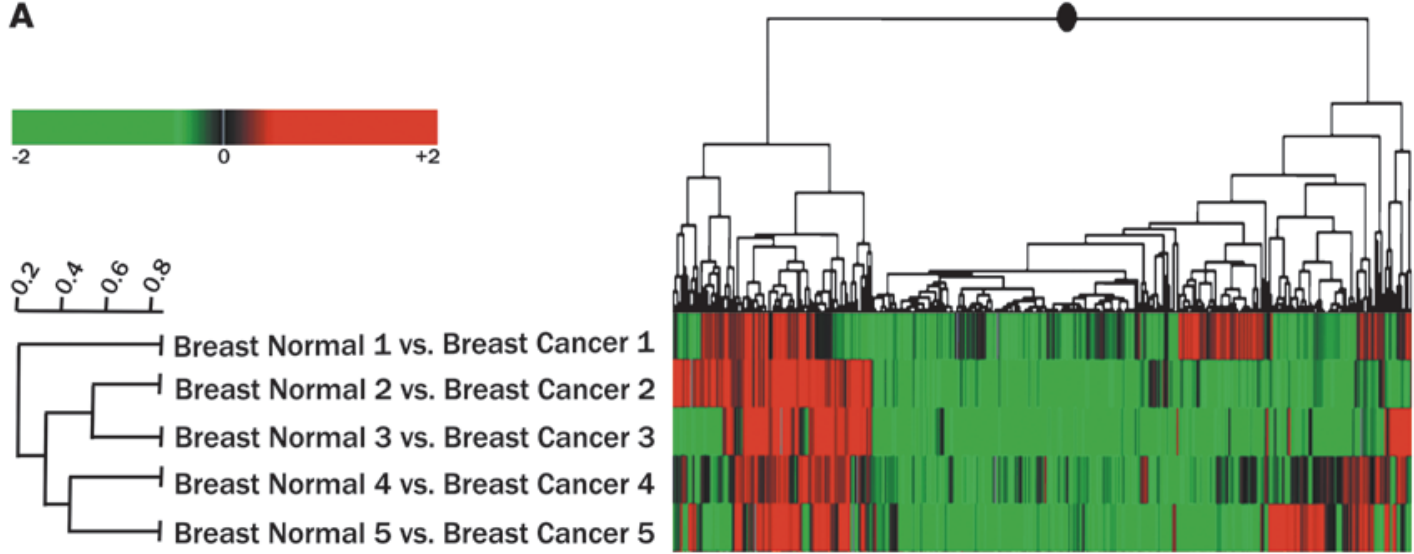

B
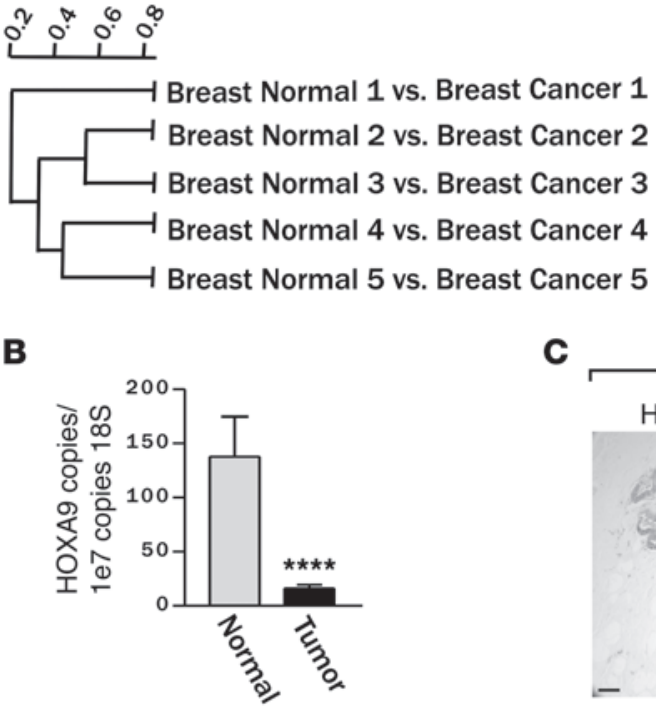

C

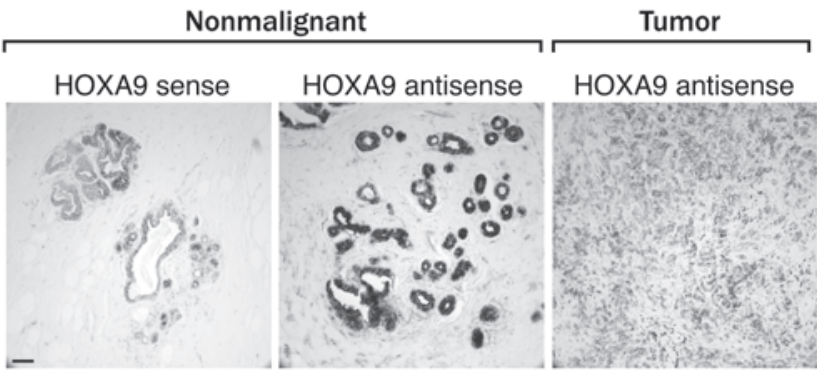

D

$2^{\circ}$ Control
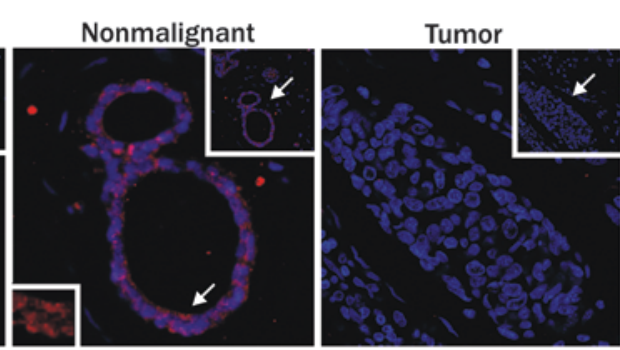

E
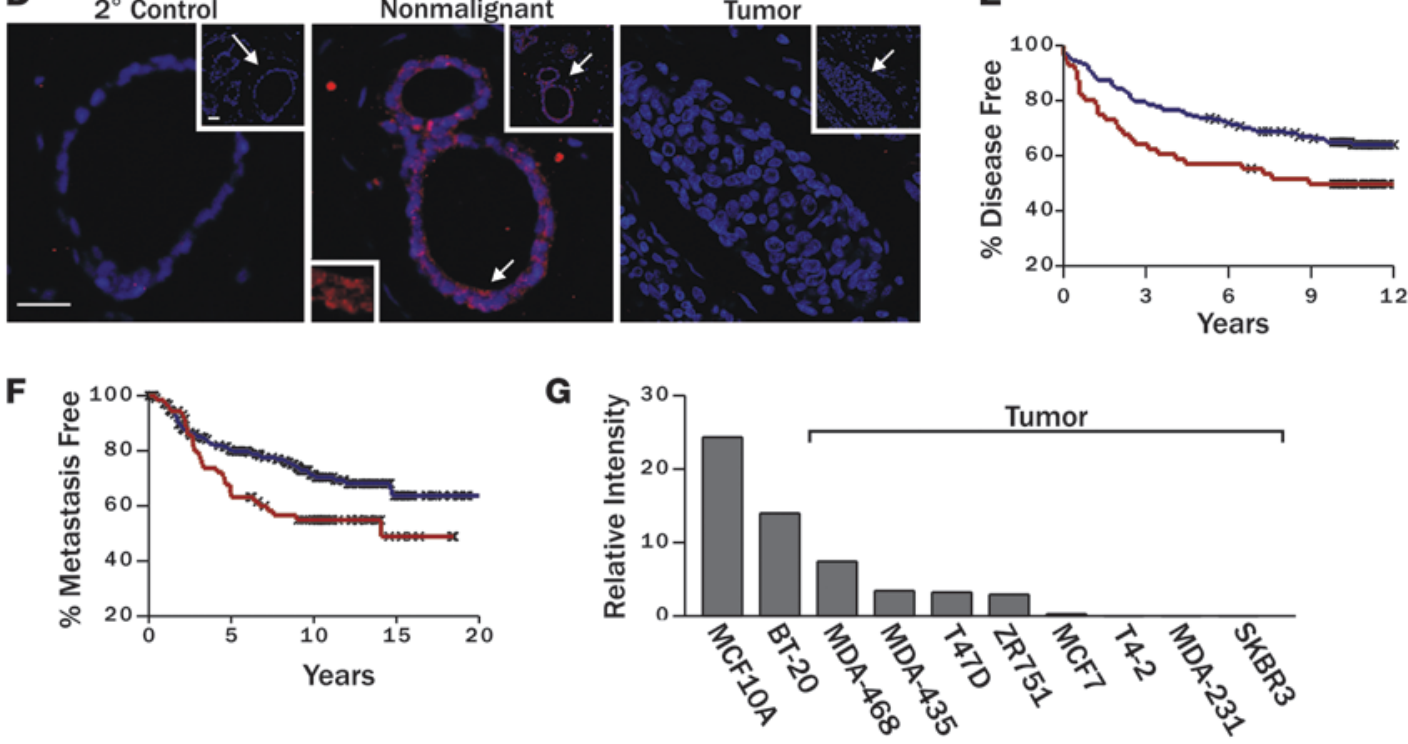

\section{Figure 1}

Breast malignancy is associated with reduced HOXA9 expression. (A) Cluster diagram of Affymetrix microarray data using Rosetta Resolver to compare gene expression profiles of matched normal mammary tissue and adjacent primary breast cancers, revealing significantly lower HOXA9 transcript levels in 4 out of 5 expression sets analyzed $(P \leq 0.01)$. (B) Levels of HOXA9 mRNA (by quantitative RT-PCR) in primary human mammary tumors $(n=47)$ compared with normal breast tissue $(n=16)$. ${ }^{* \star *} P=0.00035$. (C) In situ hybridization using a HOXA9 probe on nonmalignant $(n=4)$ or malignant $(n=6)$ mammary epithelial tissue. Scale bar: $100 \mu \mathrm{m}$. (D) Immunofluorescent staining for HOXA9 (red) and nuclei (blue) demonstrates robust cytoplasmic and nuclear localized HOXA9 protein in the epithelium of nonmalignant human breast tissue and reduced levels in breast tumors. Top right insets (original magnification, $\times 20$ ) show a broader view of the breast tissue, with arrows indicating regions blown up in the main images. The arrow in the center main image corresponds with the bottom left inset, which shows a view of HOXA9 staining (original magnification, $\times 30$ ). Scale bar: $50 \mathrm{~mm}$. The $2^{\circ}$ control shows no nonspecific staining. (E) Breast cancer patients whose tumors expressed the lowest HOXA9 level (lowest expression quartile; red line) experienced significantly reduced disease-free survival compared with all other patients in the study (blue line). An "X" is used to denote each censored sample. $P=0.025$. (F) Patients with the lowest HOXA9 levels in their tumors (lowest expression quartile; red line) also had significantly increased metastasis as a first event when compared with all other patients (blue line). An "X" is used to denote each censored sample. $P=0.02$. (G) Bar graph demonstrating relative HOXA9 gene expression levels in nonmalignant and malignant MEC lines. 


\section{Table 1}

Multivariate analysis for relapse-free and overall survival, comparing HOXA9 transcript expression and clinical characteristics in breast cancer patients

\begin{tabular}{|c|c|c|c|c|}
\hline \multirow[t]{2}{*}{ Variable } & \multicolumn{2}{|c|}{ Death } & \multicolumn{2}{|c|}{ Relapse } \\
\hline & Hazard ratio (95\% CI) & $P$ value & Hazard ratio $(95 \% \mathrm{CI})$ & $P$ value \\
\hline \multicolumn{5}{|l|}{ ER positive } \\
\hline HOXA9 & $1.185(0.91-1.541)$ & 0.208 & $1.227(1.045-1.439)$ & 0.012 \\
\hline Age (per 10-yr increment) & $0.615(0.341-1.109)$ & 0.106 & $0.527(0.331-0.842)$ & 0.007 \\
\hline Tumor size (per cm) & $1.174(0.832-1.658)$ & 0.361 & $1.177(0.896-1.546)$ & 0.242 \\
\hline \multicolumn{5}{|l|}{ Tumor grade } \\
\hline Grade 2 vs. grade 1 & $3.38(1.123-10.172)$ & 0.03 & $2.185(1.075-4.445)$ & 0.031 \\
\hline Grade 3 vs. grade 1 & $6.215(2.063-18.718)$ & 0.001 & $3.254(1.559-6.792)$ & 0.002 \\
\hline Positive vs. negative LN status & $2.366(0.995-5.628)$ & 0.051 & $2.061(1.011-4.201)$ & 0.046 \\
\hline Chemotherapy vs. no chemotherapy & $0.341(0.137-0.844)$ & 0.02 & $0.385(0.185-0.802)$ & 0.011 \\
\hline Hormonal treatment vs. no treatment & $0.753(0.239-2.373)$ & 0.628 & $0.662(0.265-1.656)$ & 0.378 \\
\hline Mastectomy vs. breast-conserving therapy & $1.283(0.678-2.428)$ & 0.444 & $1.377(0.821-2.312)$ & 0.225 \\
\hline \multicolumn{5}{|l|}{ Molecular subtype } \\
\hline Normal-like and luminal B vs. luminal A & $1.734(0.804-3.74)$ & 0.161 & $1.516(0.843-2.726)$ & 0.165 \\
\hline Basal and ERBB2+ vs. luminal A & $2.309(0.883-6.043)$ & 0.088 & $1.608(0.754-3.432)$ & 0.219 \\
\hline \multicolumn{5}{|l|}{ ER negative } \\
\hline HOXA9 & $0.394(0.181-0.857)$ & 0.019 & $0.433(0.187-1.002)$ & 0.05 \\
\hline Age (per 10-yr increment) & $0.59(0.338-1.027)$ & 0.062 & $0.552(0.314-0.973)$ & 0.04 \\
\hline Tumor size (per cm) & $1.58(1.037-2.409)$ & 0.033 & $1.49(0.958-2.319)$ & 0.077 \\
\hline Tumor grade (poorly vs. well differentiated) & $0.923(0.365-2.331)$ & 0.865 & $1.053(0.382-2.902)$ & 0.921 \\
\hline Positive vs. negative LN status & $0.64(0.209-1.954)$ & 0.433 & $0.566(0.196-1.631)$ & 0.291 \\
\hline Chemotherapy vs. no chemotherapy & $1.596(0.513-4.962)$ & 0.419 & $1.53(0.54-4.335)$ & 0.423 \\
\hline Hormonal treatment vs. no treatment & $0.423(0.083-2.154)$ & 0.3 & $0.436(0.089-2.149)$ & 0.308 \\
\hline Mastectomy vs. breast-conserving therapy & $2.1(0.926-4.759)$ & 0.076 & $2.187(0.925-5.171)$ & 0.075 \\
\hline
\end{tabular}

The analysis included the 226 ER-positive and the 69 ER-negative breast cancer patients in the Netherlands Cancer Institute data set. HOXA9 transcript expression, age, and tumor size were modeled as continuous variables. The molecular subtypes of breast cancer are not included in the model for the ER-negative cancers, as none of the tumors is categorized as the luminal A subtype, plus there is no event of death or relapse for tumors categorized as the normal-like or luminal B subtype. Statistically significant $P$ values are shown in bold.

differentiation, and BRCA1 expression is repressed following reconstituted basement membrane-induced (rBM-induced) acinar morphogenesis $(19,33-36)$. BRCA1 expression increases during embryonic mammary gland development and spikes prior to acquisition of acini polarity and pregnancy-associated lactation, consistent with the idea that BRCA1 expression is functionally linked to breast tissue differentiation $(27,32,37,38)$. If true, BRCA1 could restrict MEC growth and survival and regulate genome integrity by cooperating with molecules such as homeobox genes, which regulate tissue differentiation.

We identified HOXA9, a homeobox gene previously implicated in breast tissue differentiation (39), as a gene whose levels were reduced in breast tumors and whose reexpression promoted breast tumor morphogenesis. Although, paradoxically, HOXA9 has been characterized as a leukemic oncogene (4) and angiogenesis promoter (40), HOXA9 also plays an important role in skeletal (41), urogenital tract (42), kidney (43), and mammary gland development (39), and HOXA9 expression can be regulated by microRNAs that have been implicated in tissue differentiation (44). We found that HOXA9 promotes breast tumor cell differentiation and inhibits cancer progression by directly regulating expression of the TS gene BRCA1. Because HOXA9 is frequently methylated in breast tumors, the findings offer an alternate explanation for why BRCA1 expression is frequently lost in sporadic human breast tumors, even in the absence of genetic modification (45). They further suggest that homeobox genes could regulate tissue development and restrict tumorigenesis by modulating TS genes.

\section{Results}

Breast malignancy is associated with reduced HOXA9 expression. Expression profiling is a useful tool to identify gene expression signatures associated with patient prognosis $(46,47)$, treatment responsiveness (48-50), and risk of tumor metastasis (46, 51-53). However, because sporadic mammary tumors likely arise through input from multiple cooperating yet poorly penetrating genetic, epigenetic, and microenvironmental factors, gene profiling has proven more challenging when applied to identify breast TSs $(54,55)$. Therefore, to increase the probability of identifying a low abundance breast TS gene using this approach, we selected 5 paired sets of tumor tissue with similar phenotype. Our objective was to discover genes that have lost expression in the tumor tissues when compared with their patient-matched "normal" tissue. Because of the compelling link between developmental regulators and tumor aggression, we focused on identifying misexpressed developmental regulators $(1,56,57)$. Moreover, given the paucity of information on basal-like tumors and their recognized aggressive nature 
research article

A
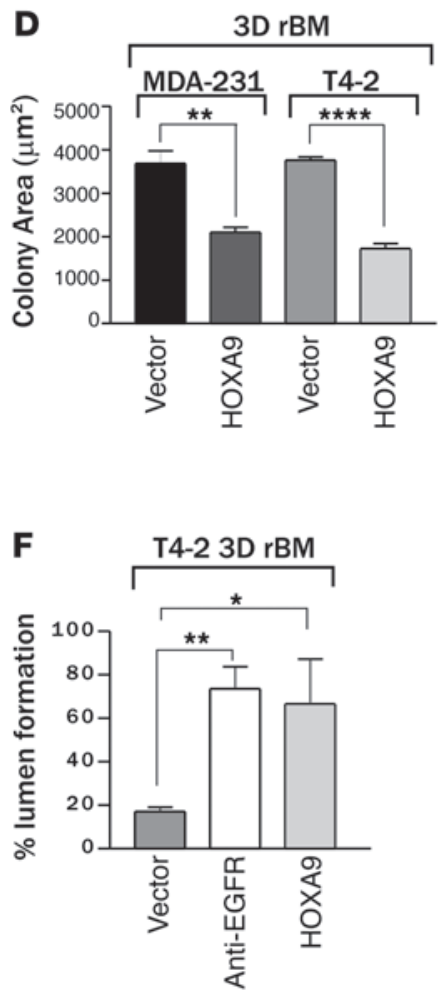

B

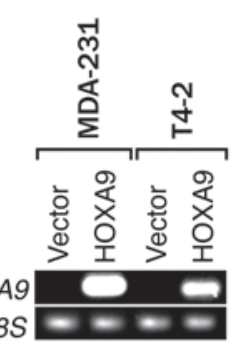

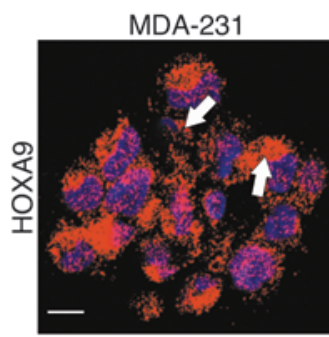

E
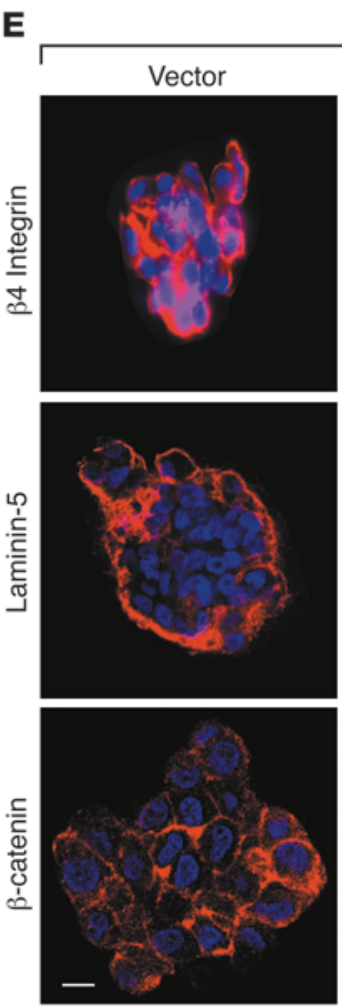

MDA-231

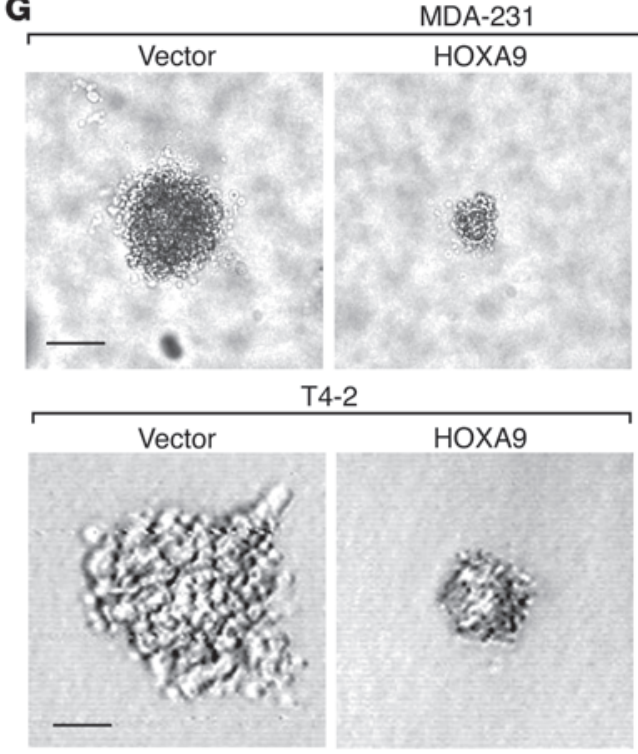

T4-2 3D rBM

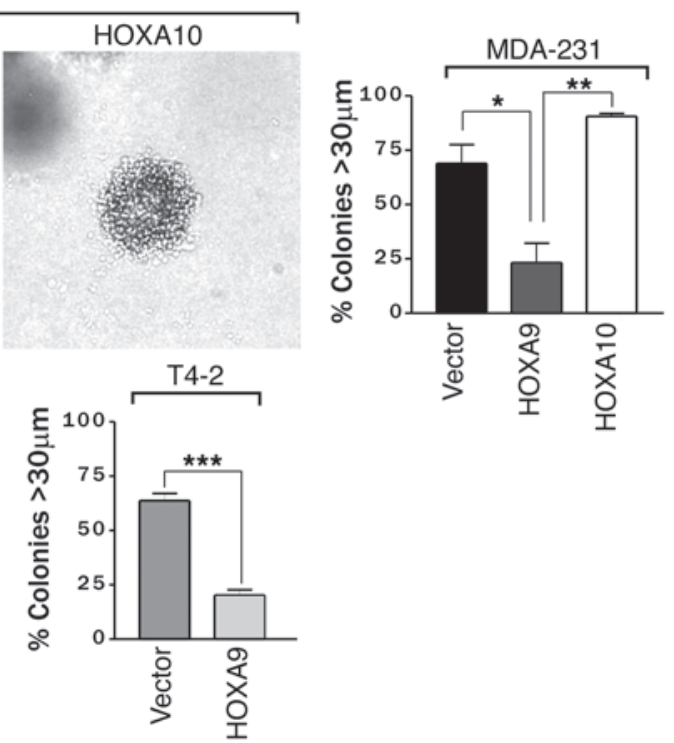

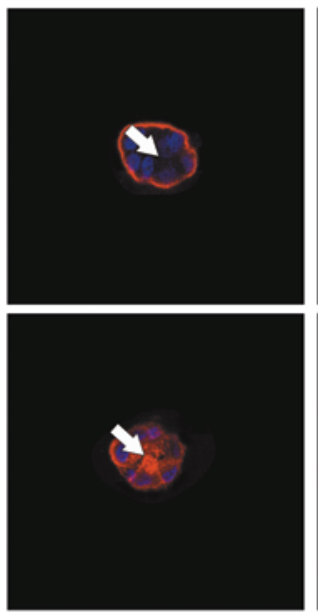

T4-2
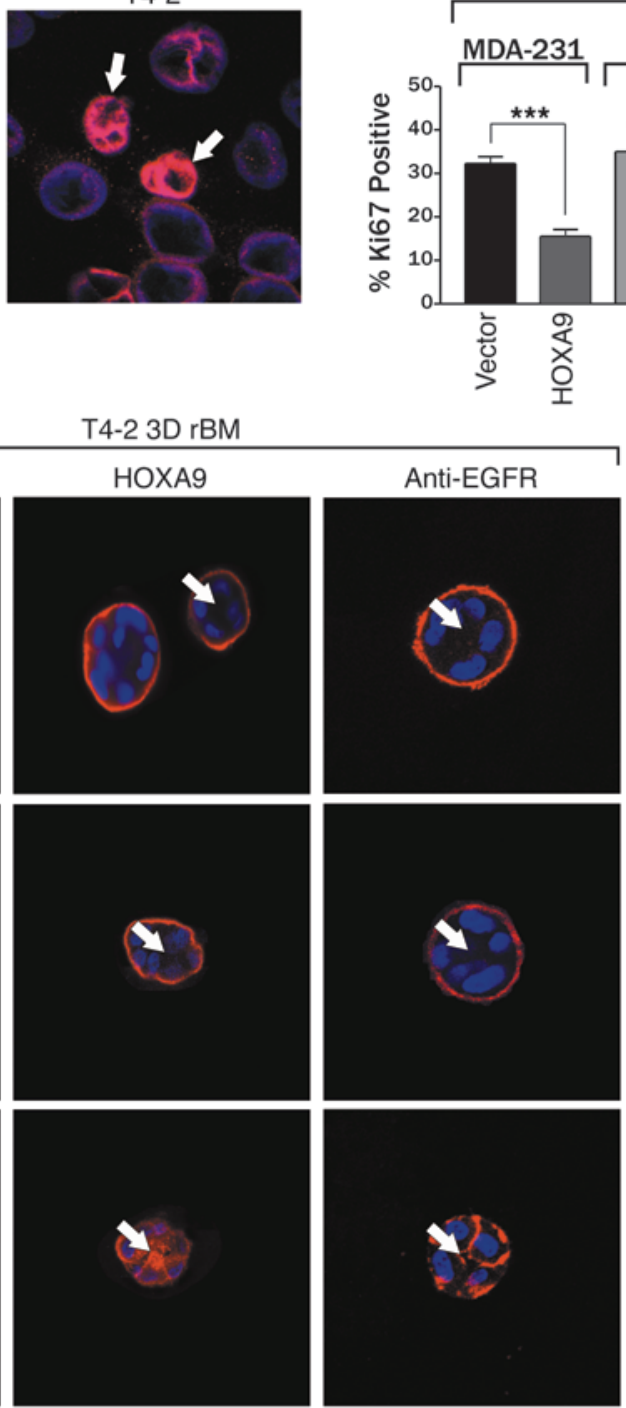

H

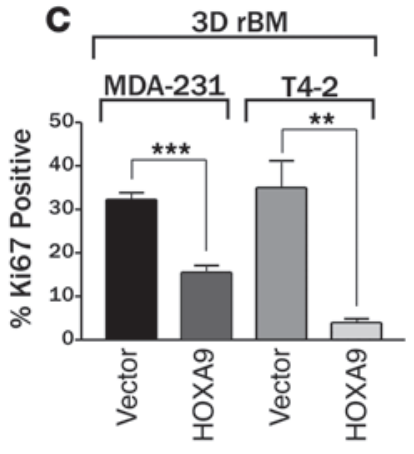

T4-2 Vector

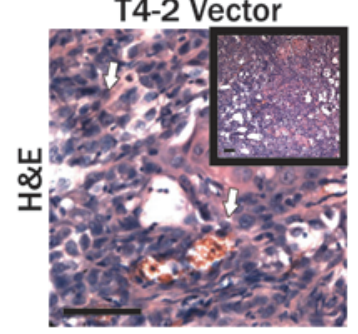

T4-2 HoxA9

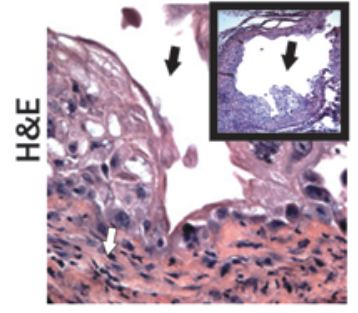




\section{Figure 2}

HOXA9 modulates the growth and survival of breast cancer cells. (A) Semiquantitative PCR gel, indicating HOXA9 mRNA levels expressed in human nonmalignant (MCF10A), metastatic (MDA-231), and transformed (T4-2) MECs. 18S rRNA was used as a control. (B) Semiquantitative PCR gel showing transgenic HOXA9 mRNA levels in MDA-231 and T4-2 cell lines. Immunofluorescence images of nuclei (blue) and FLAG-tagged HOXA9 (red) in MDA-231 and T4-2 cells. Arrows indicate localization of Flag-tagged HOXA9-positive cells. Scale bar: $10 \mu \mathrm{m}$. (C) Proliferation in MDA 231 and T4-2 cells following HOXA9 reexpression. ${ }^{\star \star} P=0.0025,{ }^{* \star} P=0.0003$. (D) Cross-sectional area of MDA-231 and T4-2 breast tumor colonies in rBM expressing either the vector or HOXA9 transgene. ${ }^{* * *} P=0.0001,{ }^{* \star} P=0.0068$. (E) Immunofluorescence images of $\beta_{4}$ integrin (red), Laminin-5 (red), $\beta$-catenin (red), and nuclei (blue) in T4-2 colonies expressing the vector or HOXA9 or phenotypically reverted acini (anti-EGFR) by inhibiting EGFR activity using tyrphostin. Arrows indicate cleared lumen. Scale bar: $10 \mu \mathrm{m}$. (F) Lumens observed in rBM-generated T4-2 colonies expressing the vector, HOXA9, or anti-EGFR phenotypically reverted acini. ${ }^{*} P=0.0188,{ }^{* \star} P=0.0076$. (G) The percentage of tumor colonies greater than $30 \mu \mathrm{m}$ in diameter, and phase contrast images of tumor colonies embedded within soft agar. Scale bar: $50 \mu \mathrm{m}$ (top panel); $20 \mu \mathrm{m}$ (bottom panel). ${ }^{\star} P=0.0221,{ }^{* \star} P=0.018,{ }^{* \star \star} P=0.0005$. (H) Highand low-magnification phase images of H\&E-stained tissue sections of control tumor (T4-2 vector) and HOXA9 reexpressing tumor (T4-2 HOXA9) xenografts. Vascularized regions of T4-2 control tumors are indicated with white arrows, and cystic regions of T4-2 HoxA9 reexpressing tumors are indicate with black arrows. Original magnification, $\times 40 ; \times 10$ (insets). Scale bar: $100 \mu \mathrm{m}$.

in younger patients, we chose samples from individuals whose ages ranged from 44 to 54 years and whose tumors were at least $1.5 \mathrm{~cm}$ in diameter (Supplemental Table 1; supplemental material available online with this article; doi:10.1172/JCI39534DS1), estrogen receptor/progesterone receptor (ER/PR) negative, and of uniformly high nuclear and histological grade.

Analysis of gene expression, using Rosetta Resolver 2D agglomerative clustering, showed significant differential expression, when comparing normal adjacent and invasive tumor tissue and identified 40 transcripts whose expression was elevated in the tumors and 115 transcripts with reduced gene expression $(P \leq 0.01$; Figure $1 \mathrm{~A}$ and Supplemental Table 2). Among the genes with reduced expression were 2 developmental HOX genes: HOXA4 (mean 3.1-fold reduction) and HOXA9 (mean 4.4-fold reduction). Findings from the boxa9-hoxb9-hoxd9 triple knockout mouse suggest that HoxA9 regulates mammary gland differentiation (39), leukemia studies implicate HoxA9 in oncogenesis (4), and in a small cohort of breast cancer patient samples HoxA9 was silenced via methylation (45); therefore, we selected HoxA9 for further study.

Quantitative RT-PCR verified that HOXA9 mRNA levels were significantly reduced in the normal compared with tumormatched clinical samples (M.A. Unger, unpublished observations) and that HOXA9 levels were significantly reduced ( $75 \%)$ in an expanded clinical cohort of ER/PR-positive and -negative, invasive, primary ductal breast carcinomas $(n=47)$, when compared with levels of transcript expressed in normal human breast ( $n=16$; Figure 1B and Supplemental Figure 1). In situ and immunohistochemical analysis confirmed that HOXA9 mRNA and HOXA9 protein were expressed in the normal breast epithelium and that levels were greatly reduced in mammary tumors (Figure 1, C and D). To explore the clinical relationship between HOXA9 levels and breast cancer clinical histopathology, we used the Oncomine Cancer Profiling Database (http://www.oncomine. org) to survey a large number of breast cancers from multiple independent studies. We found that low levels of HOXA9 expression correlate with features of aggressive disease, such as large- or high-grade tumors, late-stage disease, lymph node involvement, and distant metastasis (Supplemental Table 3). This approach not only validated our original observation that HOXA9 levels were reduced in breast cancers when compared with normal breast tissue, but they also indicated that reduced HOXA9 levels more significantly associate with tumor aggression in ER/PR-negative tumors.

To follow-up on this intriguing observation, we next analyzed publicly available gene expression data sets to look for potential associations between HOXA9 and breast cancer patient clinical outcome. First, we assessed the relationship between HOXA9 mRNA levels and relapse-free survival in 2 patient data sets that included a cohort of 227 patients, with available clinical followup information, and a second independent set of data from 295 patients $(47,58)$. We found that those patients whose tumors expressed the lowest HOXA9 levels (lowest quartile) experienced a significantly reduced relapse-free survival, regardless of ER/PR status $(P=0.025$; Figure $1 \mathrm{E})$. To determine whether low HOXA9 levels could predict distant metastasis, we also assessed the data sets from patients with early-stage breast cancer, all of whom had no evidence of distant metastasis at the time of tumor collection, for a relationship between tumor aggression and HOXA9 levels (47). Upon examination, we noted that the group of patients whose primary tumors expressed HOXA9 in the lowest quartile developed significantly more distant metastasis as a first adverse event, when compared with all other patients in the study $(P=0.02$; Figure $1 \mathrm{~F})$. A multivariate Cox proportional-hazards analysis revealed that HOXA9 significantly predicts death or disease relapse, independent of standard clinicopathological variables of breast cancers in ER-negative tumors (Table 1). In contrast, the correlation between HOXA9 expression levels and clinical outcomes was less prominent in ER-positive tumors (Table 1). These data show that HOXA9 is significantly downregulated in breast cancer and that its loss correlates with increased disease aggression.

HOXA9 modulates the growth and survival of breast cancer cells. To explore the functional relevance of HOXA9 loss to breast cancer, we examined the effect of HOXA9 reexpression on tumor cell growth, survival, and tissue differentiation. We observed that HOXA9 mRNA was abundant in the MCF10A nonmalignant human MEC line (Figure $1 \mathrm{G}$ and Figure 2A), reduced in the noninvasive, ER-positive breast cancer cell lines T47-D and MCF-7 (Figure $1 \mathrm{G}$ ), and virtually nondetectable in the ER-negative, basal-like breast cancer cell lines MDA-MB-231 (MDA-231) and HMT-3522 T4-2 (T4-2) (Figure 1G and Figure 2A) (59). We then created multiple pooled clonal populations of MDA-231 and T4-2 breast cancer cells expressing either HA- or FLAG-tagged wild-type HOXA9. Transgene expression was confirmed at the mRNA and protein

\section{Table 2}

Physical attributes of excised breast tumor cell xenografts

\begin{tabular}{lccc} 
Xenograft description & Cell mass $(\mathrm{mg})$ & Lesion & Cystic \\
T4-2 vector & $186 \pm 46.7^{\mathrm{A}}$ & $10 / 10^{\mathrm{A}}$ & $0 / 10$ \\
T4-2 HOXA9 & $82 \pm 32.3$ & $2 / 10$ & $2 / 10$ \\
\hline
\end{tabular}

${ }^{A} P<0.05$. 
A

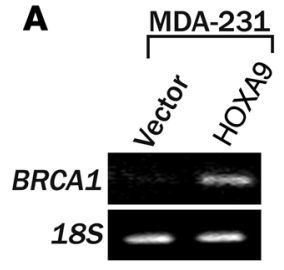

B

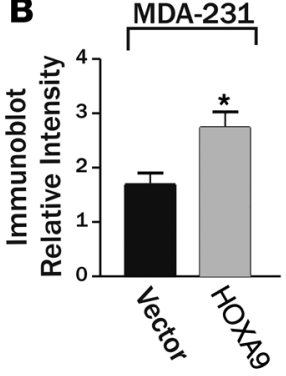

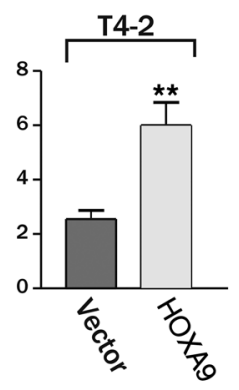

E

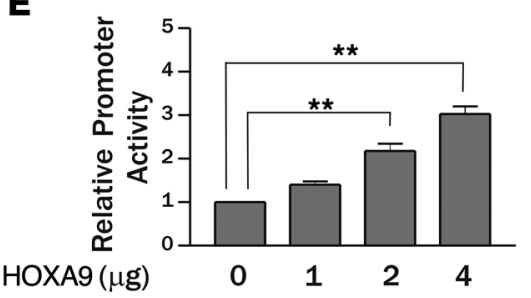

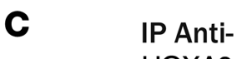

IPAnti-

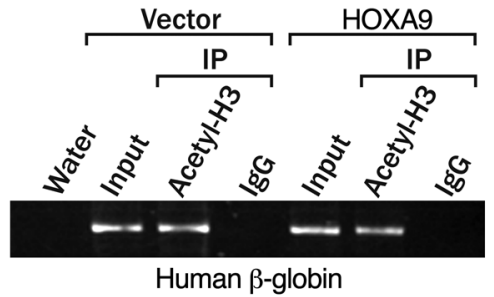

F

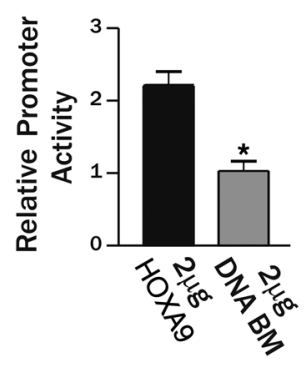

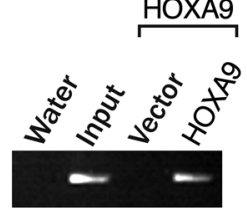

BRCA1
Human $\beta$-globin
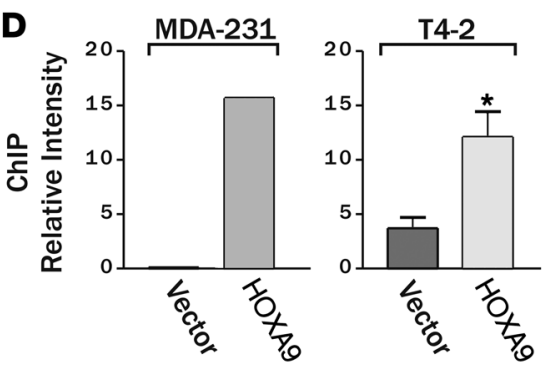

H

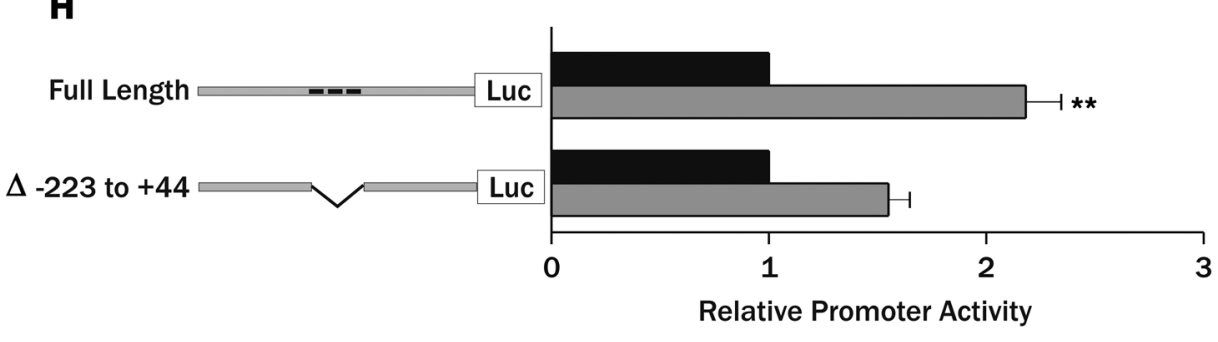

G

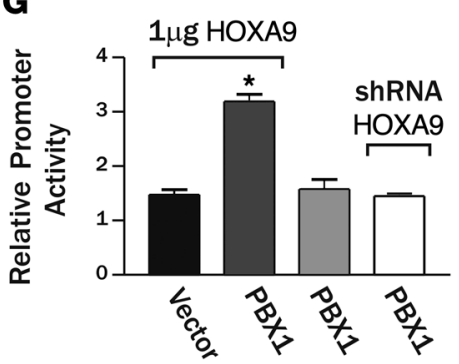

Figure 3

HOXA9 regulates BRCA1 expression. (A) Semiquantitative PCR gel, indicating increased BRCA1 expression with the reexpression of HOXA9 in MDA-231 cells. (B) Bar graph quantifying immunoblot data from multiple experiments, showing increased BRCA1 protein in MDA-231 or T4-2 breast tumor cells reexpressing HOXA9. ${ }^{*} P=0.0457,{ }^{* \star} P=0.0028$. (C) Representative gel of ChIP studies in breast cancer cells, revealing coprecipitation of HOXA9 with the BRCA1 promoter and acetylated acetyl-H3-histone with the $\beta$-globin promoter. (D) Bar graphs quantifying ChIP experiments in MDA-231 $(n=2)$ and T4-2 cells $\left({ }^{*} P=0.0178 ; n=4\right)$. (E) Luciferase reporter analysis, showing a dose-dependent increase in BRCA1 promoter activity in response to addition of wild-type HOXA9. ${ }^{* *} P=0.001$. (F) Luciferase reporter analysis, displaying loss of BRCA1 promoter activity upon addition of HOXA9 containing an N255T (DNA BM) mutation in the conserved DNA binding domain. ${ }^{*} P=0.03$ (G) Luciferase reporter analysis, indicating enhanced HOXA9-mediated BRCA1 promoter activity upon addition of PBX1 cofactor (2 $\mu \mathrm{g})$, compared with $\mathrm{Pbx} 1$ alone or $\mathrm{Pbx} 1$ cotransfected with a shRNA reducing HOXA9 expression. ${ }^{*} P=0.0259$. (H) Luciferase reporter analysis, showing a diminished responsiveness of a BRCA1 promoter construct containing a deletion in residues -223 to +44 , which contains 3 putative Hox binding sites (gray bar). Data are normalized to matched vector control (black bars). Negative numbers refer to basepairs upstream of the BRCA1 transcription start site. ${ }^{*} P=0.02$.

level (Figure 2B and Supplemental Figure 2), and the expressed protein was found to localize in both the nucleus and cytoplasm of the infected cells (Figure 2B; see white arrows), in a manner similar to endogenous HOXA9 protein expression (Figure 1, C and D). For easy visualization and manipulation, the HOXA9 transgene was expressed bi-cistronically with EGFP under the control of a tetracycline-repressible promoter (Supplemental Figure 3).

Although HOXA9 reexpression had only a marginal effect on breast tumor cell growth when the cells were propagated on tissue culture plastic (Supplemental Figure 4), we noted that upon embedment within rBM, both MDA-231 and T4-2 breast cancer cells reexpressing HOXA9 grew much slower than their respective vector-only controls, as quantified by significantly reduced Ki-67 levels (Figure 2C), and both cell lines had a decreased colony size at day 10 (Figure 2D). In fact, reexpression of HOXA9 reverted the malignant phenotype of both of the tumor cell lines toward that of a smaller, more uniform, less invasive, and more cohesive nonmalignant colony, similar to that reported previously when epidermal growth factor receptor signaling was inhibited in these cell lines (Figure 2E; see white arrows) $(60,61)$. HOXA9 reexpression had a particularly pronounced effect on the tissue morphology of the T4-2 rBM colonies, such that the HOXA9-reexpressing tumor colonies reassembled adherens junctions, as indicated by a relocalization of $\beta$-catenin to sites of cell-cell interaction, and acquired apical-basal polarity, as revealed by basal relocalization of $(\alpha 6) \beta 4$ integrin and deposition of an endogenous laminin-5 BM around the periphery of the acini (Figure 2E and Supplemental Figure 5). We also noted that HOXA9 reexpression exerted a substantial 
effect on cell viability, as revealed by the appearance of lumens within the acini (Figure 2, E and F). Soft agar assays confirmed that HOXA9 but not HOXA10 reexpression, another member of the HOXA cluster (2), influenced tumor cell survival, as shown by a significant inhibition of anchorage-independent growth and survival in both the HMT-3522 T4-2 and MDA-MB-231 tumor cells (Figure 2G, Supplemental Figure 6, and Supplemental Figure 7).

To address the functional relevance of our cell culture observations to breast cancer in vivo, we conducted xenograft studies using BalbC nu/nu mice. T4-2 breast cancer cells reexpressing HOXA9 (to levels comparable to that detected in the nonmalignant HMT3522 S-1 MECs; data not shown) failed to grow and survive when injected into the rear flanks of the BalbC $n u / n u$ mice (Figure $2 \mathrm{H}$ and Table 2). Thus, while vector control T4-2 MECs grew continuously and rapidly to form large, highly angiogenic tumors that were densely populated with actively dividing cancer cells (10 out of 10 lesions), the lesions formed by the T4-2 MECs expressing HOXA9 either completely regressed within 56 days (8 out of 10 lesions) or were highly cystic, avascular, and fibrotic (2 out of 10 lesions) (Figure $2 \mathrm{H}$ and Table 2 ). These data in conjunction with our in vitro data suggest that HOXA9 restricts tumorigenic behavior of breast cancer cells by inhibiting cell growth, survival, and invasion and by promoting tissue morphogenesis.

HOXA9 directly regulates BRCA1 expression. Hox genes are transcriptional regulators that exert their effect on cell and tissue phenotype indirectly by modulating gene expression (2). To identify putative HOXA9 targets critical for breast tumor suppression, we defined the global transcriptional profile of MDA-231 breast cancer cells before and after tetracycline-dependent HOXA9 expression (Supplemental Table 4). Among the genes upregulated after HOXA9 reexpression, we observed that the level of the breast cancer susceptibility gene BRCA1 was dramatically increased and confirmed this by semiquantitative RT-PCR (Figure 3A). Because $B R C A 1$ is a TS gene that regulates MEC growth, survival, and invasion and can modulate rBM morphogenesis, we next explored the functional relationship between HOXA9 and BRCA1 regulation $(19,32-34,38,62)$. Immunoblot analysis demonstrated that HOXA9 reexpression consistently elevated BRCA1 protein levels in MDA-231 and T4-2 breast cancer cells (Figure 3B). These results suggested that HOXA9 could repress breast tumor behavior by regulating expression of the TS gene BRCA1.

BRCA1 can be induced by multiple stimuli that might each be independently regulated by HOXA9 (27). While a number of negative regulators of BRCA1 transcription have been reported, identification of factors that directly upregulate BRCA1 expression has proven elusive (26-31). Because HOXA9 is a transcription factor, we reasoned there was a strong probability HOXA9 was regulating BRCA1 levels by directly inducing BRCA1 gene expression. Computer-assisted analysis confirmed that there were indeed several putative HOX consensus binding sites in the BRCA1 promoter. To definitively test whether HOXA9 could directly modulate BRCA1 expression, we conducted BRCA1 promoter ChIP studies using HA-tagged, exogenously expressed HOXA9 as the bait. Whereas acetyl $\mathrm{H} 3$ histone easily and repeatedly coprecipitated the $\beta$-globin promoter from both vector control- and HOXA9-expressing cell lines, we could only amplify BRCA1 promoter product above background from the breast tumor cells reexpressing the exogenous HA-tagged HOXA9 (Figure 3, C and D). Reporter assays, using regions of the BRCA1 $5^{\prime}$ promoter region containing HOXA9 consensus binding sites, confirmed basal luciferase activity could be significantly enhanced after cotransfection with increasing amounts of a wild-type HOXA9 but not with a HOXA9 expression plasmid containing an N255T mutation (DNA BM) in the conserved DNA binding domain (Figure 3, E and F) (63). Furthermore, HOXA9-dependent BRCA1 reporter induction could be significantly enhanced by addition of the HOX gene cofactor PBX1 (Figure 3G) (64). In contrast, no increase in reporter activity could be induced by PBX1 alone, PBX1 cotransfected with an shRNA knocking down HOXA9 expression, or HOXA9 cotransfection with BRCA1 luciferase promoter constructs lacking residues -223 to +44 , wherein reside putative HOXA9 consensus binding sites (Genbank U37574; Figure 3, G and H). Interestingly, site-directed mutagenesis of individual HOXA9 consensus binding sites did not significantly compromise BRCA1 promoter activity, suggesting cooperative release of tandem HOX consensus binding sites might be necessary to ablate HOXA9-dependent control of BRCA1 gene expression (Supplemental Figure 8 ). These observations are consistent with previous reports of promoter site cooperation and redundancy in other HOX-regulated genes (65-67). The findings indicate that DNA binding of HOXA9 directly regulates BRCA1 expression in breast cells.

HOXA9 regulates nonmalignant $M E C$ growth by modulating BRCA1 expression. We identified 2 independent shRNA lentiviral clones that could substantially reduce HOXA9 protein (P.M. Gilbert, unpublished observations) and HOXA9 mRNA levels in nonmalignant MCF-10A MECs (Supplemental Figure 9) to implicate HOXA9 as a TS. Loss of HOXA9 expression disrupted rBM morphogenesis, as revealed by the formation of larger invasive colonies (Figure 4B), as indicated by lack of $\beta$-catenin at sites of cellcell adherens junctions (Figure 4A, top), loss of basally polarized $(\alpha 6) \beta 4$ integrin (Figure 4A, middle), and a discontinuous endogenous laminin-5 basement membrane (Figure 4A, bottom). In addition, MCF-10A MECs with reduced HOXA9 levels exhibited an enhanced survival phenotype, as evidenced by a failure of these structures to clear their lumens (Figure 4A, see arrows; quantified in Figure 4C), and robust anchorage-independent growth and survival (Figure 4, E and F). Consistent with the notion that HOXA9 mediates these TS effects on MEC behavior by regulating BRCA1 levels, the nonmalignant MECs in which HOXA9 had been knocked down using shRNA showed a greatly blunted induction of BRCA1 transcription in response to an exogenous stress of 5 Gray irradiation (Figure 4D) (68).

To determine whether reducing BRCA1 in nontransformed MECs could similarly promote malignant behavior in these MECs, we identified 2 shRNA clones and demonstrated efficient knock down of BRCA1 protein levels (P.M. Gilbert, unpublished observations). Consistent with prior data implicating BRCA1 in breast differentiation $(19,32-34,38)$, reducing BRCA1 in the MCF-10A MECs increased cell proliferation in response to $\mathrm{rBM}$ cues (inferred by elevated colony size; Figure 4, G and $\mathrm{H}$ ), enhanced their survival as revealed by luminal filling in the rBM colonies (Figure 4G, see arrow; quantified in Figure 4I), and disrupted tissue morphogenesis (evidenced by loss of cell-cell localized $\beta$-catenin; Figure 4G). Likewise, compromising BRCA1 function in the MCF-10A cells by expressing the BRCA1 $\triangle$ exon $11 \mathrm{~b}$ mutant (Supplemental Figure 10) promoted MEC growth and survival in a 3D rBM and perturbed tissue morphogenesis (Figure 4, J-L). These observations illustrate the importance of HOXA9 and BRCA1 expression to MEC growth and survival and rBM-induced tissue morphogenesis.

HOXA9 regulates BRCA1 to repress the malignant behavior of MECs. We next manipulated the expression and function of BRCA1 and HOXA9 in breast cancer cells and assayed for effects on tumor 
A
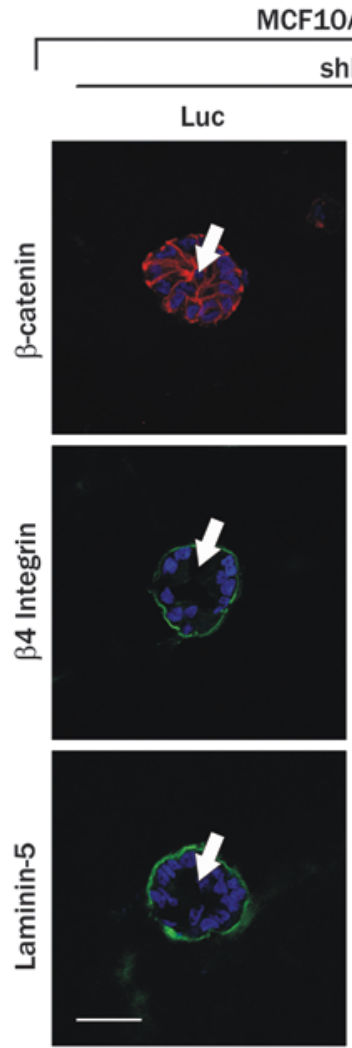

ShRNA
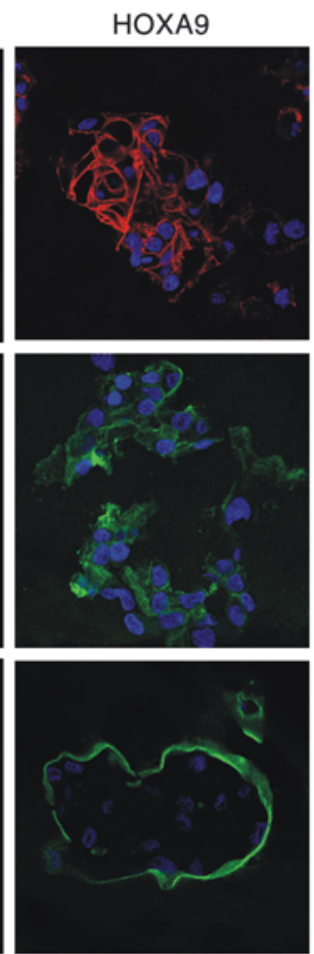

B
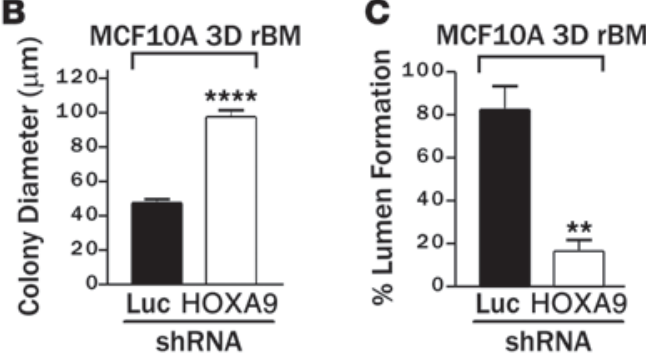

E

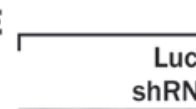

MCF10A

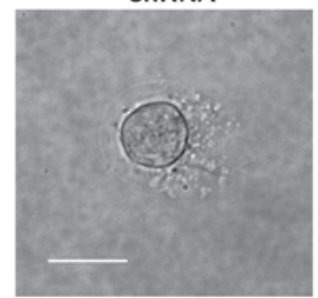

G

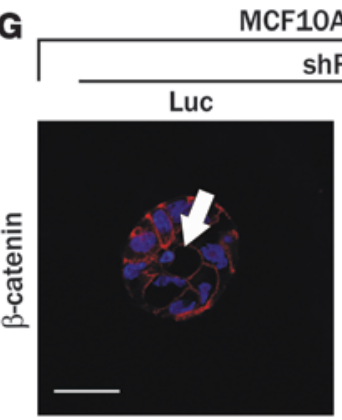

\section{RNA}

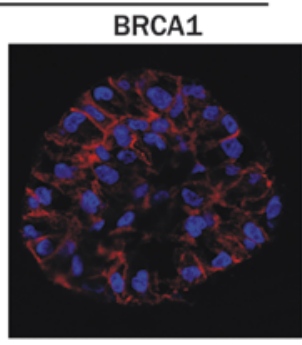

D

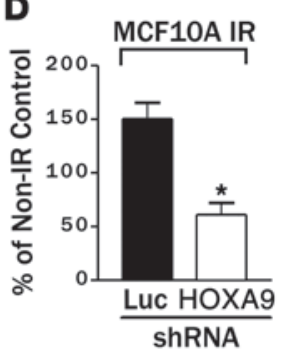

F

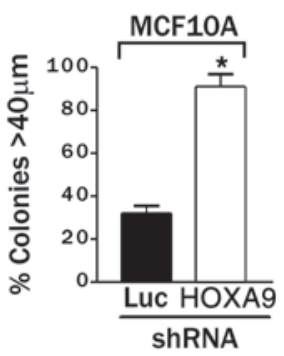

H

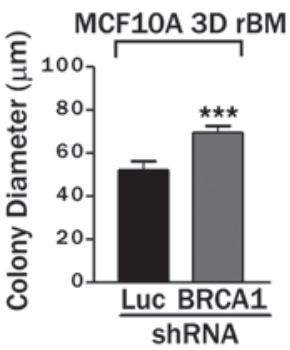

$\mathbf{L}$ MCF10A 3D rBM

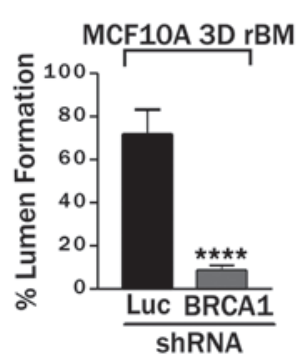

J

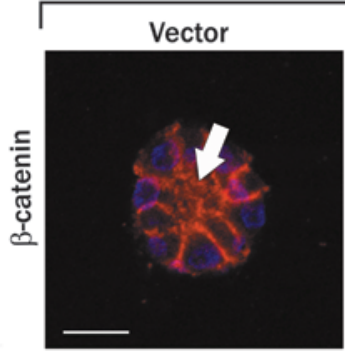

BRCA1 MT

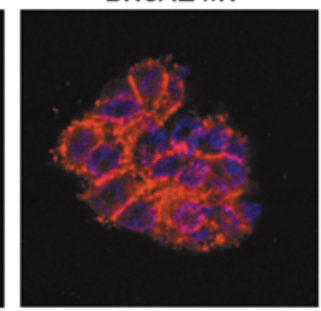

$\mathbf{K}$

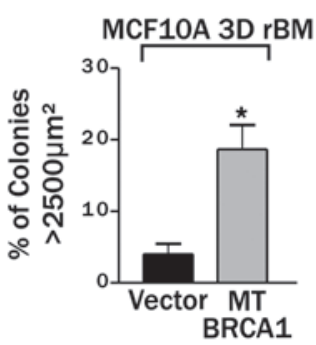

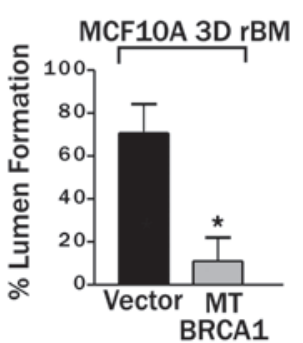

Figure 4

HOXA9 regulates nonmalignant MEC growth by modulating BRCA1 expression. (A) Immunofluorescence images of $\beta$-catenin (red), $\beta_{4}$ integrin (green), Laminin-5 (green), and nuclei (blue) in MCF-10A colonies expressing either luciferase control shRNA or shRNA-HOXA9 clone 3. Arrows indicate cleared lumen. Scale bar: $50 \mu \mathrm{m}$. (B) Colony size of MCF-10A cells cultured within a rBM and expressing reduced HOXA9 levels. ${ }^{* \star \star} P=0.0001$. (C) Lumens observed in MCF-10A colonies expressing luciferase control shRNA as compared with those with shRNA-mediated HOXA9 knockdown. ${ }^{* *} P=0.0010$. (D) The response (BRCA1 protein levels) of MCF-10A cells expressing reduced HOXA9 levels to 5 Gray irradiation compared with nonirradiated samples. ${ }^{*} P<0.001$. (E and $\left.\mathbf{F}\right)$ Phase images of tumor colonies embedded within soft agar $(\mathbf{E})$ and the percentage of colonies greater than $40 \mu \mathrm{m}$ in diameter $(\mathbf{F})$. Scale bar: $50 \mu \mathrm{m}$. ${ }^{*} P=0.001$. (G) Immunofluorescence images of $\beta$-catenin (red) and nuclei (blue) in MCF-10A colonies, expressing either luciferase control shRNA or shRNA-BRCA1 clone 5. The arrow indicates cleared lumen. Scale bar: $50 \mu \mathrm{m}$. (H) Colony size of MCF-10A cells cultured within a rBM and expressing reduced BRCA1 levels. ${ }^{* *} P=0.0024$. (I) Quantification of lumens observed in MCF-10A colonies expressing luciferase control shRNA as compared with those with shRNA-mediated BRCA1 knockdown. ${ }^{* * * *} P=0.0003$. (J) Immunofluorescence images of $\beta$-catenin (red) and nuclei (blue) in MCF-10A colonies expressing vector or mutant BRCA1 (BRCA1 MT). The arrow indicates cleared lumen. Scale bar: $10 \mu \mathrm{m}$. (K) Quantification of cross-sectional area of MCF-10A colonies in cells cultured within a rBM and coexpressing mutant BRCA1. ${ }^{*} P=0.05$. (L) Quantification of lumens observed in MCF-10A colonies expressing vector control as compared with those expressing a mutant BRCA1. ${ }^{*} P=0.05$. 
A

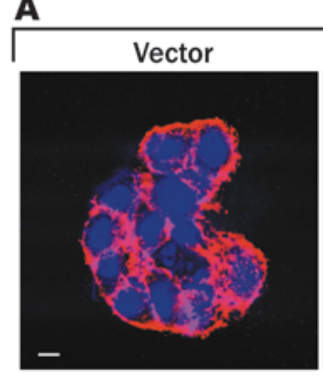

T4-2 3D rBM

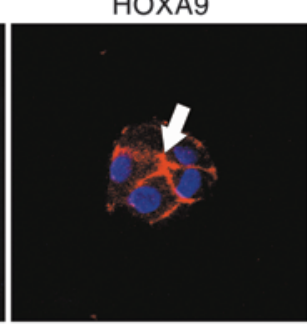

WT BRCA1

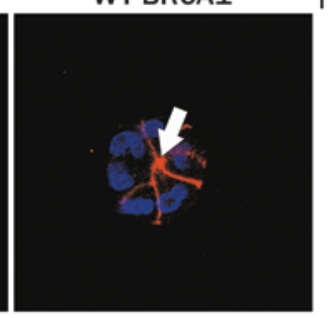

B

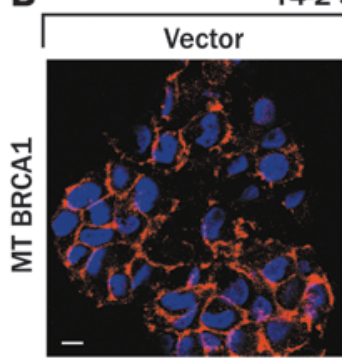

T4-2 3D rBM

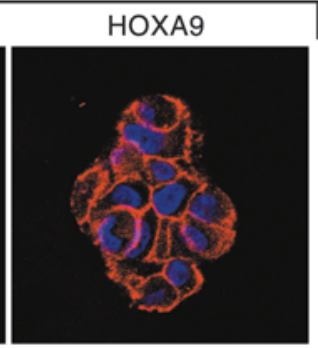

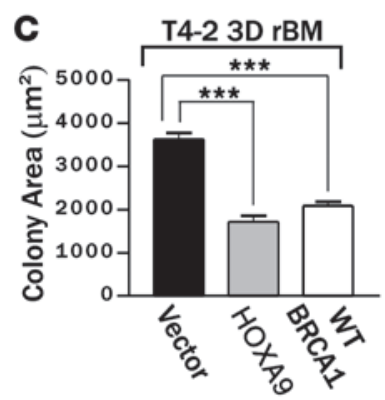

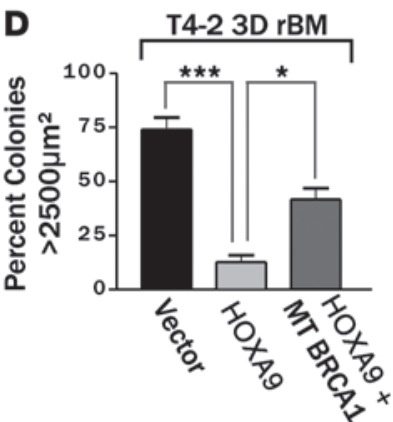

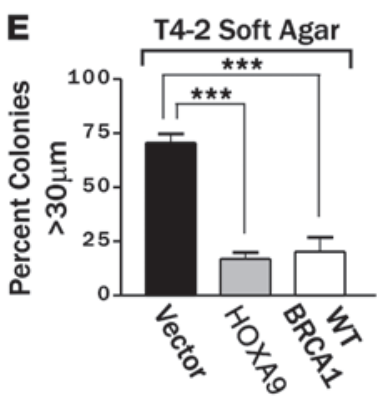

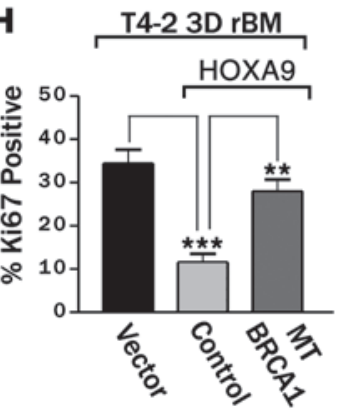

I

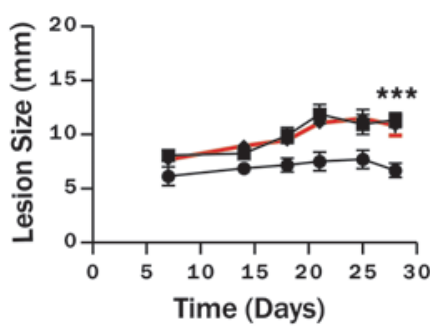

F

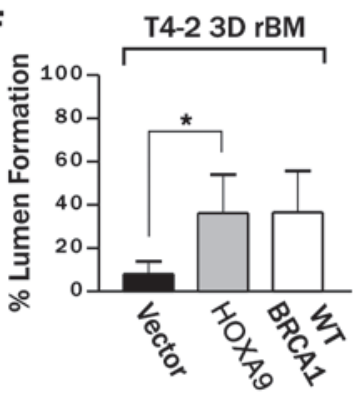

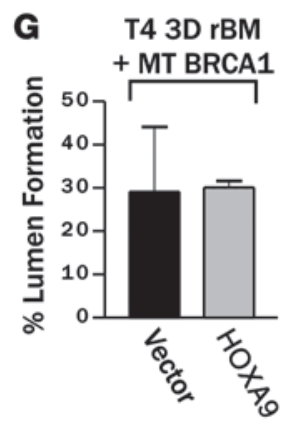

Figure 5

HOXA9 regulates BRCA1 to repress the malignant behavior of MECs. (A) Immunofluorescence images of $\beta$-catenin (red) and nuclei (blue) in T4-2 colonies reexpressing HOXA9 or BRCA1. Arrows indicate cleared lumens. Scale bar: $10 \mu \mathrm{m}$. (B) Immunofluorescence images of $\beta$-catenin (red) and nuclei (blue) in T4-2 colonies reexpressing HOXA9 alone or with a mutant BRCA1. Scale bar: $10 \mu \mathrm{m}$. (C) Quantification of colony size of T4-2 cells reexpressing HOXA9 or BRCA1. ${ }^{* * \star} P=0.001$. (D) Quantification of cross-sectional area of T4-2 colonies formed by cells reexpressing HOXA9 alone or with a mutant BRCA1. ${ }^{\star} P=0.05,{ }^{* \star *} P=0.001$. (E) Quantification of anchorage-independent growth and survival in T4-2 cells reexpressing either HOXA9 or BRCA1. ${ }^{* *} P=0.001$. (F) Quantification of the percentage of T4-2 colonies that formed lumens following the reexpression of HOXA9 or BRCA1. ${ }^{\star} P=0.0263$. (G) Quantification of lumen formation in rBM T4-2 colonies with reexpressed HOXA9, when BRCA1 function has been compromised through coexpression of a mutant BRCA1. (H) Quantification of proliferation in T4-2 cells following HOXA9 reexpression with a mutant BRCA1. ${ }^{* *} P=0.0025$, ${ }^{* *} P=0.0003$. (I) The time course of the progressive increase in xenograft size (5-30 days). Reexpression of HOXA9 in T4-2 tumor cells significantly reduced the rate of lesion expansion (filled circles) compared with the T4-2 vector controls (filled squares), which could be restored to that of T4-2 breast tumor cells if coexpressed with a mutant BRCA1 (filled triangles with red line). ${ }^{* \star} P=0.001$. (J) Lesion size (28 days) in each experimental group. ${ }^{* \star} P=0.01,{ }^{* \star *} P=0.001$.

cell behavior in culture and in vivo. Consistent with a functional link between HOXA9 and BRCA1, increasing the levels of wildtype BRCA1 in breast tumor cells significantly reduced their rBMdependent growth and normalized their survival behavior, such that they assembled colonies with cleared lumens (Figure 5A, see arrows; quantified in Figure 5F) that were similar in size and morphology to those formed by tumor cells reexpressing HOXA9 (compare in Figure 5, A, C, and F). In addition, rBM-dependent tumor colonies expressing higher wild-type BRCA1 were no longer invasive and had cell-cell localized $\beta$-catenin (Figure 5A), indicating that, analogous to HOXA9, BRCA1 also reverted the malignant phenotype of breast cells toward a normal polarized, growth-arrested acini structure (see Figure 2). Consistent with its TS function, ectopic expression of wild-type BRCA1 reduced the anchorage-independent growth and survival of breast cancer cells (Figure 5E). These data show how BRCA1 can phenocopy the TS effects of HOXA9 and can repress the growth, survival, and invasive behavior of breast tumor cells in rBM.

We compromised BRCA1 function in the T4-2 tumor cells reexpressing HOXA9 through coexpression of the $\Delta$ exon 11b BRCA1 mutant and then assayed for effects on rBM growth, survival, and colony morphology to explore the functional relationship between 
A

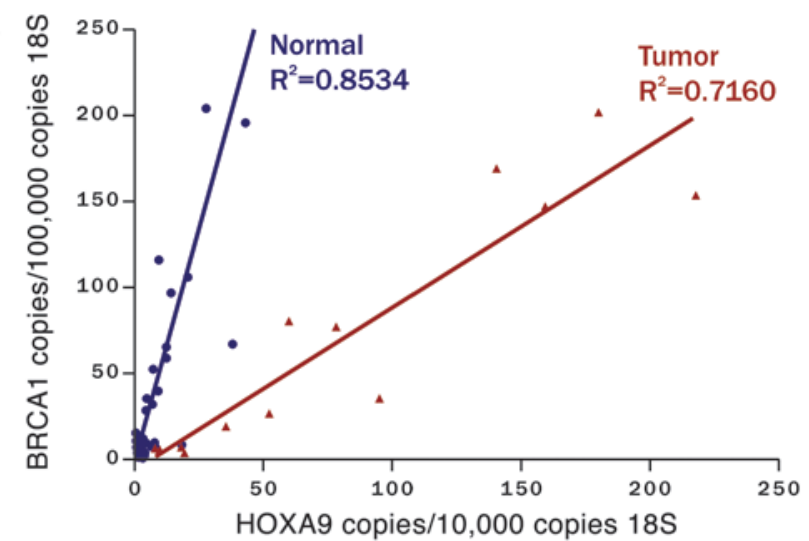

B

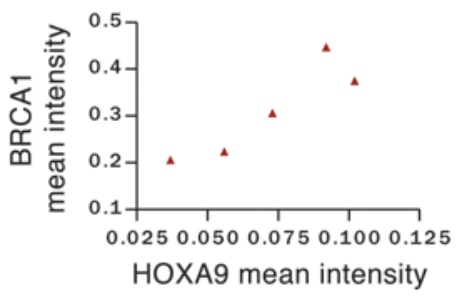

C

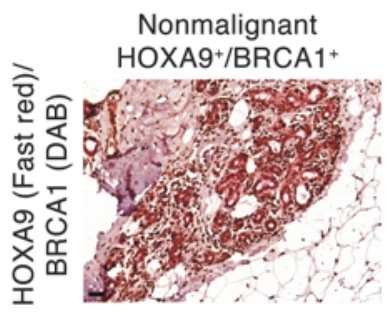

Tumor HOXA9+/BRCA1 ${ }^{+}$

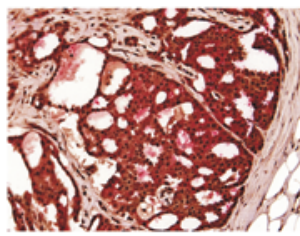

Tumor HOXA9-/BRCA1-

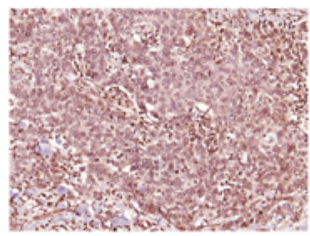

\section{Figure 6}

Clinical correlation between HOXA9 and BRCA1 expression. (A) Line graph illustrating that significant correlations exist between HOXA9 and $B R C A 1$ mRNA levels expressed in a cohort of normal (shown in blue; $P \leq 0.0001 ; r^{2}=0.8534$ ) and tumorigenic (shown in red; $P \leq 0.0001$; $r^{2}=0.7160$ ) human breast tissue specimens $(n=53)$. (B) Graph illustrating the relationship between HOXA9 and BRCA1 protein levels in human ER-/PR-/ErbB2- breast samples. (C) Immunohistochemistry showing colocalized expression of HOXA9 and BRCA1 protein in the epithelium of normal human breast tissue and HOXA9+/BRCA1+ tumor samples, as compared with HOXA9-/BRCA1- tumor samples. (HOXA9 was "stained" with Fast Red, and BRCA1 was "stained" with DAB). Scale bar: $100 \mu \mathrm{m}$.

HOXA9 and BRCA1 expression and MEC phenotype. Disrupting BRCA1 function antagonized the ability of HOXA9 to repress the malignant behavior of the T4-2 tumor cells (Figure 5B). Thus, tumor cells simultaneously expressing HOXA9 and the $\Delta$ exon $11 \mathrm{~b}$ BRCA1 mutant failed to phenotypically revert when grown within $\mathrm{rBM}$ and instead formed continuously growing, invasive, and nonpolarized colonies that lacked cell-cell localized $\beta$-catenin and cleared lumens (Figure 5, B, D, G, and H). These studies demonstrated that the ability of HOXA9 to repress the malignant behavior of MECs is functionally dependent upon BRCA1.

To address the in vivo relevance of a functional link between HOXA9 and BRCA1, we conducted xenograft studies using BalbC $n u / n u$ mice injected with human breast cancer cells, with and without HOXA9 and a functional BRCA1, and assayed tumor growth (as indicated by lesion size). Reexpression of HOXA9 in T4-2 breast cancer cells significantly reduced lesion growth rate as compared with T4-2 vector controls (Figure 5, I and J). In contrast, the HOXA9 expressing T4-2 MECs in which BRCA1 function was simultaneously compromised, through coexpression of the $\triangle$ exon11b BRCA1 mutant, grew robustly and had growth rates that were comparable to that observed in the vector control T4-2 MECs. T4-2 HOXA9 tumor cells in which BRCA1 function was compromised developed lesions that were, on average, comparable in size and morphology to those formed by the wild-type tumors (28 days; Figure 5, I and J). These data demonstrate that HOXA9 restricts the growth and survival of human breast cancer cells by regulating BRCA1 expression both in culture and in vivo.
Clinical correlation between HOXA9 and BRCA1 expression. To address the clinical relevance of a functional link between HOXA9 and BRCA1, we examined mRNA and protein expression and colocalization of HOXA9 and BRCA1 in cohorts of clinically diverse (ER/PR-positive and -negative) human breast cancers and normal tissue $(n=56)$. Quantitative RT-PCR data showed that HOXA9 correlates significantly with BRCA1 in normal $\left(r^{2}=0.8534\right.$; Figure 6A, blue line $)$ and transformed breast tissue (ER/PR positive and negative; $r^{2}=0.7160$; Figure $6 \mathrm{~A}$, red line). Quantitative immunohistochemistry of a panel of 12 triple-negative breast tumors revealed a strong positive association between nuclear HOXA9 and BRCA1 (Figure 6B and Table 3). We also noted that the epithelium of the normal breast coexpressed appreciable levels of cytoplasmic and nuclear HOXA9 and BRCA1 protein (Figure 6C, left), as did the more differentiated, triple-negative breast tumor tissues (Figure 6C, middle). In contrast, less differentiated triple-negative tumors showed complete loss of both proteins (Figure 6C, right). Interestingly, we observed that a loss in HOXA9 expression was associated with a greater reduction in BRCA1 mRNA in the breast tumor samples as compared with normal tissue (Figure 6A, compare normal to tumor). These clinical findings, together with our experimental data, are consistent with the notion that HOXA9 modulates BRCA1 expression by cooperating with multiple negative and positive regulatory factors, differentially expressed in human breast tumors, whose expression correlates with loss of ER/PR expression and tumor aggression $(20,27)$. 


\section{Discussion}

To identify developmental regulators misexpressed during malignant transformation of the breast, we used global expression profiling of microdissected breast tumors and their adjacent normal tissue. We identified the homeobox gene HOXA9, previously implicated in mammary development $(12,39)$, as a gene whose levels were significantly downregulated. We confirmed the microarray observation using RT-PCR, in situ hybridization, and immunohistochemistry and showed that HOXA9 was highly expressed in the luminal epithelium of the normal breast and that its expression was frequently decreased in a high proportion of invasive human breast tumors and breast cancer cell lines (Figure 1, $B$ and $G$ ). Bioinformatics analysis confirmed these findings and also indicated that HOXA9 loss significantly correlated with features of aggressive disease in ER/PR-negative breast tumors, including large- and high-grade tumors, late-stage disease, lymph node involvement, distant metastasis, and reduced patient survival (Figure 1, E and F, Table 1, and Supplemental Table 3). Using $2 \mathrm{ER} / \mathrm{PR} /$ ErbB2-negative, basal-like breast cancer cell lines, we then showed that reexpressing HOXA9 (but not another member of the HOXA cluster, HOXA10) inhibited tumor cell growth and survival and promoted acini morphogenesis in culture and restricted tumorigenesis in vivo. Furthermore, we showed that reducing levels of HOXA9 in nonmalignant MECs enhanced growth and survival and perturbed acini morphogenesis (Figures 2 and 4). Although investigators have previously reported that HOXA9 is methylated and that its expression is reduced in breast, lung, and ovarian cancers $(45,69,70)$, this is the first study to our knowledge to assess the clinical relationship of HOXA9 loss to solid tumors, to analyze the functional relevance of HOXA9 to the malignant behavior of MECs in culture, in vivo and in clinical specimens, and to identify a molecular mechanism for these effects. In this respect, we found that HOXA9 restricts the aggressive and malignant behavior of MECs by directly modulating BRCA1, implying that HOXA9 reduces risk to malignancy and controls tumor aggressiveness by controlling levels of an established mammary gland TS gene. These findings are consistent with the notion that developmental regulators, such as the HOX family of transcription factors, influence adult tissue homeostasis by regulating the expression and/or activity of key TS genes that regulate cell growth and survival and morphogenesis (12).

Homeobox genes regulate embryonic development and tissue patterning, and their expression is frequently perturbed and often aberrantly increased in tumors $(2,3,12,71)$. Until recently, the prevailing dogma has been that inappropriate expression of homeobox genes promotes tumor progression. Consistently, the homeobox genes that are highly expressed during early embryogenesis, that promote cell proliferation and survival, and that induce migration are those that are most often over expressed in transformed cells and tissues $(6,8,72,73)$. These are the homeobox genes that have been implicated in altered growth receptor signaling, deregulated cell cycle control, and the elevated growth and apoptosis resistance of cancer cells and that appear to regulate tumor invasion and metastasis and promote angiogenesis (40, 72, 74-76). For instance, the homeobox gene Six 1 is highly expressed during early mammary gland development, where it drives epithelial cell proliferation by modulating levels of the cell cycle gene cyclin A1 (77). Although Six1 levels are downregulated and barely detectable in the differentiated adult mammary gland, Six 1 is frequently overexpressed in aggressive breast tumors, in which it pro-
Table 3

Relationship between HOXA9 and BRCA1 nuclear protein levels in human ER-/PR-/ErbB2- breast samples

\begin{tabular}{lcc} 
Case no. & Nuclear HOXA9 & Nuclear BRCA1 \\
4309 & + & +++ \\
9667 & + & ++ \\
6073 & + & + \\
11947 & + & + \\
462 & ++ & - \\
6697 & + & - \\
8147 & - & ++ \\
8715 & - & + \\
8954 & - & + \\
6912 & - & + \\
9533 & - & - \\
2440 & - & - \\
\hline
\end{tabular}

Expression is relative between samples. -, no detectable expression; + , low expression; ++, moderate expression; +++, high expression.

motes cell growth and survival, enhances genomic instability, and promotes tumor metastasis (78). Similarly, enforced expression of the early embryonic homeobox gene Msx1, which is also often elevated in tumors, promotes the proliferation of undifferentiated stem cells, blocks the terminal differentiation of myoblasts, and downregulates expression of the myogenic differentiation factor MyoD1 to induce their malignant transformation (79-81).

It is now appreciated that homeobox genes of the ANT-C/BX-C type that control rostral-caudal patterning during embryogenesis and are abundantly expressed in differentiated tissues can repress malignancy and may function as "tumor modulators" (9, 71, 82-84). Data that support this concept exist, although, unfortunately, many findings linking homeobox gene loss with tumorigenesis are largely circumstantial $(45,69,70,79)$. At present, few methodical studies exist that clarify molecular mechanisms whereby reduced homeobox levels could restrict tumor progression and/or metastasis $(9,84)$. In this article, we present evidence that one of the posteriorly expressed homeobox genes, HOXA9, is necessary for normal MEC growth and survival and acini morphogenesis, a finding that is consistent with a previous article implicating HOXA9 in mammary gland differentiation in the mouse (Figures 2 and 4) (39). Our findings clarify these earlier observations and indicate that HOXA9 inhibits cell growth and survival and promotes morphogenesis in normal and transformed MECs by directly regulating expression of the TS gene BRCA1 (Figures 3 and 5). Our observations are consistent with and extend prior studies showing that PITX1, which is frequently downregulated in prostate, bladder, and colon cancers (6), could function as a TS by inhibiting oncogenic Ras signaling and indicating that HOXA5, which is lost in greater than $60 \%$ of mammary tumors and breast cancer cell lines, may restrict breast cancer by regulating levels of the TS p53 to alter inappropriate MEC survival $(8,73)$. Distinct from these reports, we could show that HOXA9 not only modulates BRCA1 levels but also directly binds to and regulates BRCA1 transcription and that HOXA9-dependent BRCA1 induction is markedly enhanced during tissue remodeling and following exposure of breast cells to an exogenous stress, consistent with a role for BRCA1 in cell cycle regulation and the DNA damage response $(85,86)$. Intriguingly, HoxC8 was shown to bind directly to and regulate the mammalian 
homolog of lethal giant larvae TS gene; however, to date no functional data exist to clarify the relevance of this relationship (7). In the present studies, we not only showed that HOXA9 directly regulates BRCA1 transcription, but we demonstrated that HOXA9dependent BRCA1 expression is critical for the growth, survival, and morphogenesis activity of HOXA9 in culture and for its TSlike activity in vivo (Figures 4 and 5). We also provided additional evidence that this relationship has clinical relevance (Figures 5 and 6). Indeed, our findings argue that loss of HOXA9 is a critical determinant of tumor aggression, especially in ER/PR-negative breast tumors, which likely harbor additional modifications that negatively modulate BRCA1 levels. Our findings emphasize the importance of examining the role of homeobox family members as critical regulators of normal tissue differentiation and homeostasis and illustrate the potential permissive role of HOXA9 loss in tumor progression and aggressiveness and as a potential regulator of treatment responsiveness.

In contrast to our observation that HOXA9 restricts the growth, survival, and malignant behavior of breast epithelial cells, it is well known that HOXA9 plays an essential role in normal myeloid lineage development, because it promotes expansion of the stem cell pool and inhibits differentiation (87). Consistently, increased expression or activation of HOXA9 in myeloid stem cells is causally linked to acute myeloid leukemia (88), and enforced expression of HOXA9 in myeloid cells, due to chromosome translocation or overexpression of its regulator MLL, drives transformation (4). Furthermore, elevated HOXA9 expression induces angiogenesis by regulating growth, migration, and invasion of endothelial cells (40). Interestingly, neither myeloid cells nor human endothelial cells ectopically overexpressing HOXA9 upregulate $B R C A 1$ transcript levels, thereby offering one likely explanation for the strikingly different phenotypic consequences of HOXA9 expression between MECs versus lymphocytes and endothelial cells (P.M. Gilbert, unpublished observations) (89, 90). This observation is consistent with previous studies indicating that homeobox target genes are cell and tissue specific. The data are also in accord with results showing that the expression profile and gene targets of each HOX factor depend upon the complement of cofactors present in each cell and the extracellular microenvironment the cell resides within (91). Thus, while HOXA5 can induce p53 expression in MECs, sustained HOXA5 failed to modulate p53 in endothelial cells and instead induced Thrombospondin- $2(8,92)$. Such findings serve to illustrate the urgency of conducting comparative functional studies of homeobox gene regulation in different tissues and stress the relevance of tissue context as a key regulator of cellular behavior. The work also underscores the importance of considering tissue-specific gene regulation in order to understand cancer pathogenesis as well as to identify tissue-specific treatments.

BRCA1 is either lost or mutated in many cases of familial breast cancer $(27,93,94)$. Nevertheless, BRCA1 expression is also frequently reduced in sporadic breast cancers, and gene methylation and silencing only account for a subset of these sporadic tumors $(27,93)$. This means that other parameters and factors that are altered during breast carcinogenesis likely exist to regulate tissuespecific levels of BRCA1 $(20,27)$. Indeed, the BRCA1 promoter is a highly complex, bidirectional transcriptional unit, with multiple binding motifs, and it is subject to dynamic interactions between its promoter and terminator regions. Its activity can be modulated by multiple generic and tissue-specific factors, including 53BP1,
E2F proteins, and GABP- $\alpha / \beta$, and conditions, including stress, hypoxia, growth factors, and estrogens $(95,96)$. However, despite these findings, very few factors have been shown to bind to and directly modulate BRCA1 expression and, of these, most have been negative regulators. Furthermore, there is a paucity of evidence to link these BRCA1 regulators to defined BRCA1-dependent phenotypes. Metastasis-associated tumor antigen 1 (MTA1) has been implicated in the transcriptional repression of BRCA1 and in abnormal centrosome number and chromosomal instability (30), and E2F4 and the pocket proteins p130/p107 bind the BRCA1 promoter and repress its basal transcription, thereby regulating cell growth (97). In these studies, we demonstrate that HOXA9 directly and positively modulates BRCA1 transcription, thereby restricting the abnormal growth, survival, and stress response of breast cancer cells and nonmalignant MECs in culture and in vivo. This relationship has clinical relevance because loss of HOXA9 significantly predicts tumor aggression in ER/PR-negative cancers. In this respect, HOXA9 correlated significantly with BRCA1 mRNA and BRCA1 protein levels in breast tissues, in which we observed that reduced HOXA9 expression was associated with a more profound reduction of BRCA1 in tumor cells and that loss of HOXA9 and BRCA1 was strongly associated with a less differentiated phenotype in triple-negative breast tumors. Because basal-like tumors also frequently express "BRCA1 repressors," these results argue that HOXA9 likely cooperates with other intrinsic or acquired factors to modulate BRCA1 expression in breast cancers. The fact that expression profiling did not reveal reduced $B R C A 1$ transcripts in the 4 primary tumors with reduced HOXA9 expression is not surprising (Supplemental Table 2). In general, expression levels of BRCA1 are below the detection sensitivity of Affymetrix arrays, and thus transcript level changes would not normally be noted. Instead, BRCA1 expression is tightly linked to cell cycling and the presence of damaged DNA (98), and we observed a robust induction of BRCA1 in response to HOXA9 most predominantly during tissue remodeling (J.K. Mouw, unpublished observations) or after exposure to an exogenous stress (Figure 4D). Thus, because the HOXA9 promoter is frequently methylated and HOXA9 levels are often reduced in invasive breast tumors, and we showed that HOXA9 is often lost in breast tumors, our data offer an attractive explanation for why BRCA1 expression could be so frequently lost in sporadic human breast tumors, even in the absence of genetic aberrations, promoter methylation, or haploinsufficiency.

Intriguingly, not only did we find that HOXA9-dependent BRCA1 expression restricts tumor progression by inhibiting MEC growth and survival, but we also observed that elevated HOXA9 and BRCA1 levels restored cell-cell adhesions and normalized acini morphogenesis (Figures 2 and 5). These findings are consistent with previous reports that showed that loss of BRCA1 compromises the ability of nonmalignant MCF10A MECs to undergo morphogenesis into polarized acinar structures in a 3D rBM assay $(19,35,36)$ and are consistent with data indicating that BRCA1 is critical for lumen formation in primary murine MECs (38). Indeed, during mammary gland remodeling, BRCA1 levels peak prior to tight junction assembly and tissue-specific differentiation, and they decline to barely detectable levels during lactation $(27,37,38)$. Consistently, recent studies in which BRCA1 was overexpressed in the epithelium of the murine breast showed that there was a moderate increase in lobular alveolar differentiation in the mammary gland, consistent with accelerated development, and these mice also showed resistance to mutagen-induced mammary neoplasia. In contrast, age-matched 
mice expressing a mutated BRCA1 morphologically resembled animals at mid-pregnancy, consistent with increased proliferation and secondary branching, and these mice showed enhanced DMBAinduced transformation (99). Our findings raise the intriguing possibility that some HOX genes, particularly those expressed late during development and those that are expressed in differentiated tissues such as HOXA9, might regulate growth, survival, and invasion to modulate body plan patterning during development, by regulating levels of TSs that control these orchestrated processes. In this respect, HOXA9 plays an important role in skeletal (41), urogenital tract (42), kidney (43), and mammary gland development (39), and HOXA9 expression can be regulated by microRNAs that have been implicated in tissue differentiation (44).

\section{Methods}

Substrates, antibodies, and pharmacological reagents. The materials used were as follows: Commercial EHS matrix (Matrigel; Collaborative Research) for the rBM assays; Vitrogen (Vitrogen 100, Inamed Biomaterials; bovine skin collagen I), $3 \mathrm{mg} / \mathrm{ml}$, for coating culture dishes; and Cellagen AC-5, 0.5\% (ICN Biomedical Inc.) for morphogenesis assays. Primary antibodies used were as follows: actin, clone AC-40 (Sigma-Aldrich); BRCA1, clone Ab-1 (Oncogene); $\beta$-Catenin, clone 14 (BD Biosciences - Transduction Laboratories); $\beta_{4}$ integrin, clone 3E1 (Invitrogen); FLAG, clone M2 (Sigma-Aldrich); HA.11, clone 16B12 (Covance Research Products); HA, clone Y-11 (Santa Cruz Biotechnologies Inc.); acetyl H3 histone, rabbit polyclonal (Upstate); Ki-67 clone 35 (BD Biosciences - Transduction Laboratories); HOXA9 (N-20), goat polyclonal (Santa Cruz Biotechnologies Inc.); HOXA9, rabbit polyclonal (a gift from T. Nakamura, Japanese Foundation for Cancer Research, Tokyo, Japan); HOXA10 rabbit polyclonal (Abcam); and laminin 5 (a gift from M.P. Marinkovich, Stanford University School of Medicine, Stanford, California, USA) (100). Secondary antibodies used were Alexa Fluor 488- and 555-conjugated polyclonal anti-rabbit and anti-mouse IgGs (Molecular Probes) and HRP-conjugated polyclonal rabbit and anti-mouse IgGs (Amersham Pharmacia). Pharmaceutical reagents included Tyrphostin AG $1478(100 \mu \mathrm{M}$; DMSO) (Calbiochem); $p$-Nitrophenyl phosphate disodium salt hexahydrate (Fisher Scientific); and D-Luciferin, potassium salt (Biotium).

cDNA, lentiviral and retroviral, and shRNA constructs and vectors. Please refer to the Supplemental Methods section for a detailed description of constructs used.

Cell culture. The HMT-3522 S-1 and T4-2 MECs were grown and manipulated in $2 \mathrm{D}$ and $3 \mathrm{D}$, and the T4-2s were phenotypically reverted exactly as described previously $(60,101)$. MDA-MB-231, MCF-7, and MCF10A cells were cultured according to manufacturer's recommendations (ATCC) and grown in 3D as described previously (102). BT-20, MDA-MB-468, MDAMB-435, T47D, ZR751, and SK-BR-3 cells were cultured according to manufacturer's recommendations (ATCC).

Retroviral and lentiviral infections. Amphotrophic retrovirus was produced (103), and retroviral supernatant was harvested and used directly to spin infected cells, followed by antibiotic-induced selection with puromycin $(0.5 \mu \mathrm{g} / \mathrm{ml}$ media) or neomycin $(100 \mu \mathrm{g} / \mathrm{ml}) 72$ hours after infection (104). Lentiviral particles were produced, harvested, and used to infect target cells as previously described (105).

Soft agar assay. Anchorage-independent growth was assessed using a soft agar assay (60). In brief, 25,000 cells in $1.5 \mathrm{ml} 0.35 \%$ agarose containing $1 \mathrm{X}$ growth media were overlaid with $1.5 \mathrm{ml} 0.5 \%$ agarose containing $1 \mathrm{X}$ growth media, and colonies larger than $30 \mu \mathrm{m}$ in diameter were scored positive after 14 days.

In vivo studies. All experiments were performed in accordance with the guidelines of Laboratory Animal Research at the University of Pennsylvania. Fourweek-old BalbC $n u / n u$ mice were subcutaneously injected in the rear flanks $\left(5 \times 10^{6}\right.$ cells/injection, together with Matrigel), and palpable lesions were detected, measured, and monitored biweekly for 56 days (Instant read-out digital calipers; Electron Microscopy Sciences). At experiment termination, mice were sacrificed, and lesions were dissected, measured, macroscopically analyzed, fixed in $4 \%$ paraformaldehyde, and paraffin embedded, and H\&E sections were evaluated for histopathological evidence of tumor phenotype.

Immunofluorescence. Immunofluorescence analysis of cells grown in $2 \mathrm{D}, 3 \mathrm{D}$, and paraffin-embedded tissues was performed as previously described $(101,102)$.

Proliferation. Cell proliferation was measured by calculating the percentage of Ki-67-labeled nuclei and quantified as previously described (106).

Immunoblotting. Equal amounts of cell protein lysate (Laemmli; BCA; Pierce) were separated on reducing SDS-PAGE gels, transferred to nitrocellulose or PVDF membrane, and probed with primary antibody. Bands were visualized and quantified using a Fujifilm Gel Documentation system, in conjunction with HRP-conjugated secondary antibodies and ECL-Plus (Amersham Pharmacia). For the irradiation response of MCF-10A cells, cells were irradiated (5 Gray) and lysed 24 hours later.

ChIP. ChIP assays were performed according to the manufacturer's directions (Upstate). In brief, proteins were cross-linked to chromatin (formaldehyde; 1\%), cells were lysed, and the chromatin was sheared (sonication; Misonix Ultrasonic). HA-tagged HOXA9/DNA fragments were immunoprecipitated (overnight; at $4^{\circ} \mathrm{C}$ ) using polyclonal anti-HA (clone $\mathrm{Y}-11$ ) or polyclonal anti-HOXA9, with polyclonal anti-acetyl $\mathrm{H} 3$ histone serving as a positive control and isotype-matched IgG serving as a negative control. Protein/DNA complexes were captured (Protein A agarose beads), washed (6-10 times), and eluted from beads, and cross-links were reversed ( $\mathrm{NaCl}$ and phenol/chloroform extraction). DNA was ethanol precipitated and used directly for PCR reactions. To amplify a human BRCA1 promoter fragment from anti-HOXA9 ChIP experiments we used the following primers: forward, 5'-GATGGGACCTTGTGGAAGAA-3', and reverse, 5'-ACGACCAAACCAACACCAAT- $3^{\prime}$. To amplify the human $\beta$-globin gene (107) from anti-acetyl $\mathrm{H} 3$ histone ChIP experiments we used the following primers: forward, 5'-ATCTTCCTCCCACAGCTCCT-3', and reverse, 5'-TTTGCAGCCTCACCTTCTTT-3'. Bar graph data is normalized to an IgG control ChIP.

$B R C A 1$ reporter assay. Luciferase $B R C A 1$ gene reporter assays were conducted in 293 cells by transient transfection and normalizing transfection efficiency, by quantifying SEAP expression using a MRX microplate reader (Dynex Technologies) 36 hours after transfection, as previously described (108). Forty-eight hours after transfection, cells were lysed (25 mM glycylglycine, 2 mM EGTA, pH 8.0, 1\% Triton X-100, 1 mM DTT, pH 7.8), aliquots of lysate were diluted (1:5) in assay buffer (25 mM glycylglycine, 2 mM EGTA, pH 8.0, $10 \mathrm{mM} \mathrm{MgSO}_{4}, 2.2 \mathrm{mM}$ ATP, $0.275 \mathrm{mM}$ Acetyl CoA, $1 \mathrm{mM}$ DTT, $\mathrm{pH}$ 7.8), transferred to a Microfluor plate (Thomas Scientific), and mixed with equal quantity of luciferin buffer ( $25 \mathrm{mM}$ glycylglycine, $2 \mathrm{mM}$ EGTA, $\mathrm{pH}$ 8.0, $10 \mathrm{mM} \mathrm{MgSO}_{4}, 1 \mathrm{mM}$ DTT, 0.55 mM luciferin, $\mathrm{pH}$ 7.8). Light emission from the reaction was detected using a Microtiter plate luminometer (Dynex Technologies) in conjunction with Revelation software. Experiments were quantified as the fold change over appropriate control conditions.

Morphometric analysis. Colony size and morphology in 3D rBM was assessed at indicated times, essentially as previously described $(101,102)$. Briefly, cell-cell integrity within a 3D MEC acinus was considered intact when $\beta$-catenin staining was localized and continuous around the periphery of all cells. A 3D MEC acinus was considered properly polarized when $\beta_{4}$ integrin and laminin were expressed continuously and exclusively around the basal surface of the acinar structure.

Expression profiling. Please see the Supplemental Methods section for a detailed description of the expression profiling.

Semiquantitative PCR. Purified total RNA $(2.0 \mu \mathrm{g})$ was reverse transcribed, using random primers (Amersham Biosciences), and resultant samples were serially diluted $1: 10,1: 100$, and $1: 1,000$ for subsequent $P C R$ reactions. 
An initial PCR was performed to amplify the 18S rRNA subunit, together with a standard curve to determine cDNA copy number for each sample. Primer sequences were as follows: $18 \mathrm{~S}$ rRNA, forward, $5^{\prime}$-CGGCTACCACATCCAAGGAA-3', and $18 S$ rRNA, reverse, 5'-GCTGGAATTACCGCGGCT- $3^{\prime}$. Corrected cDNA concentrations were calculated and a second PCR reaction was performed in which equal amounts of cDNA were added to primers specific for HOXA9. The primer sequences used to amplify HOXA9 cDNA were 5'-GCTTGTGGTTCTCCTCCAGT-3' and 5'-CCAGGGTCTGGTGTTTTGTA-3'. These primers cross the exon 1-2 boundary and thus should not amplify contaminating genomic DNA. The primer sequences used to amplify BRCA1 cDNA were 5'-GGAACTAACCAAACGGAGCA-3' and $5^{\prime}$-TAGGTTTCTGCTGTGCCTGA- 3 '. The primer sequences used to amplify HOXA10 cDNA were 5'-TATCCCACAACAATGTCATGCTC-3' and 5'-GTCGCCTGGAGATTCATCAGGA-3'.

Quantitative real-time PCR. Total RNA was reverse transcribed using random primers (Amersham Biosciences), and 18S rRNA primers were used to control for cDNA concentration in a separate PCR reaction for each sample (see above for sequences). Primers used to amplify HOXA9 exon 2, using the LightCycler apparatus (Roche Diagnostics), are listed above. LightCycler Fast Start DNA Master SYBR Green Mix (Roche) was added to each PCR reaction along with cDNA and 1 pmol primer in a total volume of $10 \mu \mathrm{l}$. The primers and conditions used to amplify the BRCA 1 cDNA junction between exon 12 and 13 were previously described (109).

In situ hybridization. Sense and antisense riboprobes against HOXA9 were generated as previously described (83). Digoxygenin-labeled probes were prepared using T7 or SP6 RNA polymerase (Roche). Paraffin-embedded human breast tissue was hybridized with $800 \mathrm{ng} / \mathrm{ml}$ of probe as previously described (83). Six normal and four invasive ductal carcinoma human breast samples were examined.

Immunohistochemistry. Formalin-fixed, paraffin-embedded human breast tissue sections, lacking any patient-identifying information, were obtained with IRB approval from the University of Pennsylvania and the Huntsman Cancer Institute Tissue Resource and Acquisition Core Facility with patient consent. Sections were deparaffinized and rehydrated through 3 concentrations of alcohol and incubated in $3 \% \mathrm{H}_{2} \mathrm{O}_{2}$ for 15 minutes to block endogenous peroxidase. Antigen retrieval was carried out in $0.1 \mathrm{M}$ citrate buffer, $\mathrm{pH} 6.0$, at $95^{\circ} \mathrm{C}$ for 20 minutes, followed by 20 minutes at room temperature. Nonspecific binding was blocked using PBS containing $1 \%$ BSA and $5 \%$ goat serum for 30 minutes. Sections were probed with anti-HOXA9 (1:200) and anti-BRCA1 antibodies. Biotinylated secondary antibody and $\mathrm{ABC}$ reagent were used as directed (Vector Laboratories). Fast red (HOXA9) and DAB (BRCA1) were used as chromogens (Vector Laboratories). Sections were counterstained with Mayer's hematoxylin (SigmaAldrich). Please refer to the supplemental method section for information regarding multispectral image acquisition and analysis. Six normal and four invasive ductal carcinoma samples were examined.

Bioinformatics analysis. Please refer to the Supplemental Methods section for a detailed description of the bioinformatics analysis.

Statistics. We used InStat software (Graphpad) to conduct the statistical analysis of our data. Unless otherwise stated, 2-tailed Student $t$-tests were used for simple significance testing, and 2-tailed Pearson tests were used for correlation analysis (mean \pm SEM of 3-5 independent experiments). $P$ values of less than 0.05 were considered to be significant. Unless otherwise noted, $n=3$.

\section{Acknowledgments}

We thank P. Marinkovich for the BM165 mAb, H. Blau for the Hermes-HRS-puro-IRES-EGFP construct, C. Largman for the PRC-CMV-HOXA9 construct, F. Rauscher for the HA-tagged wild-type and $\triangle$ exon $11 \mathrm{~b}$ BRCA1 constructs, and L.A. Chodosh for the pGL2-BRCA1 luciferase construct. This work was supported by NIH grants R01-CA078731 and U54CA143836; DOD grants DAMD17-01-1-0368, DAMD17-03-1-0396, and W81XWH-051-330; DOE grant A107165; CIRM grant RS1-00449 (to V.M. Weaver); Komen grant DISS0 402407 (to P.M. Gilbert); DOD grant BC062562 (to J.K. Mouw); and by the Huntsman Cancer Foundation/Hunstman Cancer Institute (to A.L. Welm).

Received for publication April 13, 2009, and accepted in revised form February 8, 2010.

Address correspondence to: Valerie M. Weaver, UCSF, Center for Bioengineering and Tissue Regeneration, Department of Surgery, 513 Parnassus Avenue, S1364C-0456, San Francisco, California 94143, USA. Phone: 415.476.3826; Fax: 415.476.3985; E-mail: Valerie.Weaver@ucsfmedctr.org.

Penney M. Gilbert's present address is: Baxter Laboratories in Stem Cell Biology, Department of Microbiology and Immunology, Institute for Stem Cell Biology and Regenerative Medicine, Stanford University School of Medicine, Stanford, California, USA.
1. Chen H, Sukumar S. HOX genes: emerging stars in cancer. Cancer Biol Ther. 2003;2(5):524-525.

2. Grier DG, Thompson A, Kwasniewska A, McGonigle GJ, Halliday HL, Lappin TR. The pathophysiology of HOX genes and their role in cancer.J Pathol. 2005;205(2):154-171.

3. Samuel S, Naora H. Homeobox gene expression in cancer: insights from developmental regulation and deregulation. Eur J Cancer. 2005;41(16):2428-2437.

4. Nakamura T, et al. Fusion of the nucleoporin gene NUP98 to HOXA9 by the chromosome translocation $\mathrm{t}(7 ; 11)(\mathrm{p} 15 ; \mathrm{p} 15)$ in human myeloid leukaemia. Nat Genet. 1996;12(2):154-158.

5. Ford HL, Kabingu EN, Bump EA, Mutter GL, Pardee AB. Abrogation of the $\mathrm{G} 2$ cell cycle checkpoint associated with overexpression of HSIX1: a possible mechanism of breast carcinogenesis. Proc Natl Acad Sci U S A. 1998;95(21):12608-12613.

6. Kolfschoten IG, et al. A genetic screen identifies PITX1 as a suppressor of RAS activity and tumorigenicity. Cell. 2005;121(6):849-858.

7. Tomotsune D, Shoji H, Wakamatsu Y, Kondoh $\mathrm{H}$, Takahashi N. A mouse homologue of the Drosophila tumour-suppressor gene 1(2)gl controlled by Hox-C8 in vivo. Nature. 1993;365(6441):69-72.
8. Raman V, et al. Compromised HOXA5 function can limit 553 expression in human breast tumours. Nature. 2000;405(6789):974-978.

9. Carrio M, Arderiu G, Myers C, Boudreau NJ. Homeobox D10 induces phenotypic reversion of breast tumor cells in a three-dimensional culture model. Cancer Res. 2005;65(16):7177-7185.

10. Lewis MT. Homeobox genes in mammary gland development and neoplasia. Breast Cancer Res. 2000;2(3):158-169.

11. Duverger O, Morasso MI. Role of homeobox genes in the patterning, specification, and differentiation of ectodermal appendages in mammals. J Cell Physiol. 2008;216(2):337-346.

12. Chen H, Sukumar S. Role of homeobox genes in normal mammary gland development and breast tumorigenesis. J Mammary Gland Biol Neoplasia. 2003;8(2):159-175.

13. Friedman LS, et al. Confirmation of BRCA1 by analysis of germline mutations linked to breast and ovarian cancer in ten families. Nat Genet. 1994;8(4):399-404.

14. Starita LM, Horwitz AA, Keogh MC, Ishioka C, Parvin JD, Chiba N. BRCA1/BARD1 ubiquitinate phosphorylated RNA polymerase II. J Biol Chem.
2005;280(26):24498-24505

15. Fan W, et al. BRCA1 regulates GADD45 through its interactions with the OCT-1 and CAAT motifs. J Biol Chem. 2002;277(10):8061-8067.

16. Aprelikova O, Pace AJ, Fang B, Koller BH, Liu ET. BRCA1 is a selective co-activator of 14-3-3 sigma gene transcription in mouse embryonic stem cells. J Biol Chem. 2001;276(28):25647-25650.

17. Rosen EM, Fan S, Pestell RG, Goldberg ID. BRCA1 gene in breast cancer.J Cell Physiol. 2003;196(1):19-41.

18. Burga LN, et al. Altered proliferation and differentiation properties of primary mammary epithelial cells from BRCA1 mutation carriers. Cancer Res. 2009;69(4):1273-1278.

19. Furuta S, Jiang X, Gu B, Cheng E, Chen PL, Lee WH. Depletion of BRCA1 impairs differentiation but enhances proliferation of mammary epithelial cells. Proc Natl Acad Sci U S A. 2005; 102(26):9176-9181.

20. Thompson ME, Jensen RA, Obermiller PS, Page DL, Holt JT. Decreased expression of BRCA1 accelerates growth and is often present during sporadic breast cancer progression. Nat Genet. 1995;9(4):444-450.

21. Honrado E, Benitez J, Palacios J. The molecular 
pathology of hereditary breast cancer: genetic testing and therapeutic implications. Mod Pathol. 2005;18(10):1305-1320.

22. Sorlie T, et al. Repeated observation of breast tumor subtypes in independent gene expression data sets. Proc Natl Acad Sci U S A. 2003;100(14):8418-8423.

23. Turner N, Tutt A, Ashworth A. Hallmarks of 'BRCAness' in sporadic cancers. Nat Rev Cancer. 2004;4(10):814-819.

24. Wei M, et al. BRCA1 promoter methylation in sporadic breast cancer is associated with reduced BRCA1 copy number and chromosome 17 aneusomy. Cancer Res. 2005;65(23):10692-10699.

25. Futreal PA, et al. BRCA1 mutations in primary breast and ovarian carcinomas. Science. 1994;266(5182):120-122.

26. Baldassarre G, et al. Negative regulation of BRCA1 gene expression by HMGA1 proteins accounts for the reduced BRCA1 protein levels in sporadic breast carcinoma. Mol Cell Biol. 2003;23(7):2225-2238.

27. Mueller CR, Roskelley CD. Regulation of BRCA1 expression and its relationship to sporadic breast cancer. Breast Cancer Res. 2003;5(1):45-52.

28. Antonova L, Mueller CR. Hydrocortisone downregulates the tumor suppressor gene BRCA1 in mammary cells: a possible molecular link between stress and breast cancer. Genes Chromosomes Cancer. 2008;47(4):341-352.

29. Beger C, et al. Identification of Id 4 as a regulator of BRCA1 expression by using a ribozyme-librarybased inverse genomics approach. Proc Natl Acad Sci US A. 2001;98(1):130-135.

30. Molli PR, Singh RR, Lee SW, Kumar R. MTA1-mediated transcriptional repression of BRCA1 tumor suppressor gene. Oncogene. 2008;27(14):1971-1980.

31. Thakur S, et al. Regulation of BRCA1 transcription by specific single-stranded DNA binding factors. Mol Cell Biol. 2003;23(11):3774-3787.

32. Marquis ST, et al. The developmental pattern of Brca1 expression implies a role in differentiation of the breast and other tissues. Nat Genet. 1995;11(1):17-26.

33. Xu X, et al. Conditional mutation of Brca1 in mammary epithelial cells results in blunted ductal morphogenesis and tumour formation. Nat Genet. 1999;22(1):37-43.

34. Kubista M, Rosner M, Kubista E, Bernaschek G, Hengstschlager M. Brca1 regulates in vitro differentiation of mammary epithelial cells. Oncogene. 2002;21(31):4747-4756.

35. O'Connell FC, Martin F. Laminin-rich extracellular matrix association with mammary epithelial cells suppresses Brca1 expression. Cell Death Differ. 2000;7(4):360-367.

36. Miralem T, Avraham HK. Extracellular matrix enhances heregulin-dependent BRCA1 phosphorylation and suppresses BRCA1 expression through its C terminus. Mol Cell Biol. 2003;23(2):579-593.

37. Magdinier F, Dalla Venezia N, Lenoir GM, Frappart L, Dante R. BRCA1 expression during prenatal development of the human mammary gland. Oncogene. 1999;18(27):4039-4043.

38. Murtagh J, McArdle E, Gilligan E, Thornton L, Furlong F, Martin F. Organization of mammary epithelial cells into 3D acinar structures requires glucocorticoid and JNK signaling. J Cell Biol. 2004;166(1):133-143.

39. Chen F, Capecchi MR. Paralogous mouse Hox genes, Hoxa9, Hoxb9, and Hoxd9, function together to control development of the mammary gland in response to pregnancy. Proc Natl Acad Sci U S A. 1999;96(2):541-546.

40. Bruhl T, Urbich C, Aicher D, Acker-Palmer A, Zeiher AM, Dimmeler S. Homeobox A9 transcriptionally regulates the EphB4 receptor to modulate endothelial cell migration and tube formation. Circ Res. 2004;94(6):743-751.

41. Chen F, Capecchi MR. Targeted mutations in hoxa-
9 and hoxb-9 reveal synergistic interactions. Dev Biol. 1997;181(2):186-196.

42. Taylor HS, Vanden Heuvel GB, Igarashi P. A conserved Hox axis in the mouse and human female reproductive system: late establishment and persistent adult expression of the Hoxa cluster genes. Biol Reprod. 1997;57(6):1338-1345.

43. Dintilhac A, Bihan R, Guerrier D, Deschamps S, Pellerin I. A conserved non-homeodomain Hoxa9 isoform interacting with CBP is co-expressed with the 'typical' Hoxa9 protein during embryogenesis. Gene Expr Patterns. 2004;4(2):215-222.

44. Shen WF, Hu YL, Uttarwar L, Passegue E, Largman C. MicroRNA-126 regulates HOXA9 by binding to the homeobox. Mol Cell Biol. 2008;28(14):4609-4619.

45. Reynolds PA, et al. Tumor suppressor p16INK4A regulates polycomb-mediated DNA hypermethylation in human mammary epithelial cells. J Biol Chem. 2006;281(34):24790-24802.

46 . van ' $t$ Veer LJ, et al. Gene expression profiling predicts clinical outcome of breast cancer. Nature. 2002;415(6871):530-536.

47. van de Vijver MJ, et al. A gene-expression signature as a predictor of survival in breast cancer. $N$ Engl J Med. 2002;347(25):1999-2009.

48. Sotiriou C, et al. Gene expression profiles derived from fine needle aspiration correlate with response to systemic chemotherapy in breast cancer. Breast Cancer Res. 2002;4(3):R3.

49. Perou CM, et al. Molecular portraits of human breast tumours. Nature. 2000;406(6797):747-752.

50. Petty RD, et al. Tumor transcriptome reveals the predictive and prognostic impact of lysosomal protease inhibitors in non-small-cell lung cancer. J Clin Oncol. 2006;24(11):1729-1744.

51. Livasy CA, et al. Phenotypic evaluation of the basallike subtype of invasive breast carcinoma. Mod Pathol. 2006;19(2):264-271.

52. Ramaswamy S, Ross KN, Lander ES, Golub TR. A molecular signature of metastasis in primary solid tumors. Nat Genet. 2003;33(1):49-54.

53. Weigelt B, Glas AM, Wessels LF, Witteveen AT, Peterse JL, van't Veer LJ. Gene expression profiles of primary breast tumors maintained in distant metastases. Proc Natl Acad Sci U S A. 2003; 100(26):15901-15905.

54. Kurose K, Hoshaw-Woodard S, Adeyinka A, Lemeshow S, Watson PH, Eng C. Genetic model of multi-step breast carcinogenesis involving the epithelium and stroma: clues to tumour-microenvironment interactions. Hum Mol Genet. 2001;10(18):1907-1913.

55. Comings DE, Gade-Andavolu R, Cone LA, Muhleman D, MacMurray JP. A multigene test for the risk of sporadic breast carcinoma. Cancer. 2003; 97(9):2160-2170.

56. Mani SA, et al. Mesenchyme Forkhead 1 (FOXC2) plays a key role in metastasis and is associated with aggressive basal-like breast cancers. Proc Natl Acad Sci U S A. 2007;104(24):10069-10074.

57. Mani SA, et al. The epithelial-mesenchymal transition generates cells with properties of stem cells. Cell. 2008;133(4):704-715.

58. Ivshina AV, et al. Genetic reclassification of histologic grade delineates new clinical subtypes of breast cancer. Cancer Res. 2006;66(21):10292-10301.

59. Kenny PA, et al. The morphologies of breast cancer cell lines in three-dimensional assays correlate with their profiles of gene expression. Mol Oncol. 2007;1(1):84-96.

60. Wang F, et al. Reciprocal interactions between beta1integrin and epidermal growth factor receptor in three-dimensional basement membrane breast cultures: a different perspective in epithelial biology. Proc Natl Acad Sci U S A. 1998;95(25):14821-14826.

61 . Wang F, et al. Phenotypic reversion or death of cancer cells by altering signaling pathways in threedimensional contexts. J Natl Cancer Inst. 2002; 94(19):1494-1503.
62. Rajan JV, Wang M, Marquis ST, Chodosh LA. Brca2 is coordinately regulated with Brca1 during proliferation and differentiation in mammary epithelial cells. Proc Natl Acad Sci US A. 1996;93(23):13078-13083.

63. Thakur S, Croce CM. Positive regulation of the BRCA1 promoter. J Biol Chem. 1999;274(13):8837-8843.

64. Moens CB, Selleri L. Hox cofactors in vertebrate development. Dev Biol. 2006;291(2):193-206.

65. Maconochie M, Nonchev S, Morrison A, Krumlauf R. Paralogous Hox genes: function and regulation. Annu Rev Genet. 1996;30:529-556.

66. Chang CP, Brocchieri L, Shen WF, Largman C, Cleary ML. Pbx modulation of Hox homeodomain amino-terminal arms establishes different DNAbinding specificities across the Hox locus. Mol Cell Biol. 1996;16(4):1734-1745.

67. Beachy PA, Varkey J, Young KE, von Kessler DP, Sun BI, Ekker SC. Cooperative binding of an Ultrabithorax homeodomain protein to nearby and distant DNA sites. Mol Cell Biol. 1993;13(11):6941-6956.

68. Xu B, Kim S, Kastan MB. Involvement of Brca1 in S-phase and G(2)-phase checkpoints after ionizing irradiation. Mol Cell Biol. 2001;21(10):3445-3450.

69. Choi JS, et al. Comparative genomic hybridization array analysis and real-time PCR reveals genomic copy number alteration for lung adenocarcinomas. Lung. 2006;184(6):355-362.

70. Wu Q, et al. DNA methylation profiling of ovarian carcinomas and their in vitro models identifies HOXA9, HOXB5, SCGB3A1, and CRABP1 as novel targets. Mol Cancer. 2007;6:45.

71. Abate-Shen C. Deregulated homeobox gene expression in cancer: cause or consequence? Nat Rev Cancer. 2002;2(10):777-785.

72. Hartwell KA, Muir B, Reinhardt F, Carpenter AE, Sgroi DC, Weinberg RA. The Spemann organizer gene, Goosecoid, promotes tumor metastasis. Proc Natl Acad Sci U S A. 2006;103(50):18969-18974.

73. Stasinopoulos IA, Mironchik Y, Raman A, Wildes F, Winnard P Jr, Raman V. HOXA5-twist interaction alters p53 homeostasis in breast cancer cells. J Biol Chem. 2005;280(3):2294-2299.

74. Boudreau N, Andrews C, Srebrow A, Ravanpay A, Cheresh DA. Induction of the angiogenic phenotype by Hox D3. J Cell Biol. 1997;139(1):257-264.

75. Myers C, Charboneau A, Boudreau N. Homeobox B3 promotes capillary morphogenesis and angiogenesis. J Cell Biol. 2000;148(2):343-351.

76. Boudreau NJ, Varner JA. The homeobox transcription factor Hox D3 promotes integrin alpha5beta1 expression and function during angiogenesis. J Biol Chem. 2004;279(6):4862-4868.

77. Coletta RD, et al. The Six1 homeoprotein stimulates tumorigenesis by reactivation of cyclin A1. Proc Natl Acad Sci U S A. 2004;101(17):6478-6483.

78. Coletta RD, Christensen KL, Micalizzi DS, Jedlicka P, Varella-Garcia M, Ford HL. Six1 overexpression in mammary cells induces genomic instability and is sufficient for malignant transformation. Cancer Res. 2008;68(7):2204-2213.

79. Ruhin-Poncet B, et al. Msx and dlx homeogene expression in epithelial odontogenic tumors. J Histochem Cytochem. 2009;57(1):69-78.

80. Lee H, Habas R, Abate-Shen C. MSX1 cooperates with histone $\mathrm{H} 1 \mathrm{~b}$ for inhibition of transcription and myogenesis. Science. 2004;304(5677):1675-1678.

81. Song K, Wang Y, Sassoon D. Expression of Hox7.1 in myoblasts inhibits terminal differentiation and induces cell transformation. Nature. 1992; 360(6403):477-481.

82. Ma L, Teruya-Feldstein J, Weinberg RA. Tumour invasion and metastasis initiated by microRNA-10b in breast cancer. Nature. 2007;449(7163):682-688.

83. Myers C, Charboneau A, Cheung I, Hanks D, Boudreau N. Sustained expression of homeobox D10 inhibits angiogenesis. Am J Pathol. 2002;161(6):2099-2109.

84. Abate-Shen C, et al. Nkx3.1; Pten mutant mice 
develop invasive prostate adenocarcinoma and lymph node metastases. Cancer Res. 2003; 63(14):3886-3890.

85. Mullan PB, Quinn JE, Harkin DP. The role of BRCA1 in transcriptional regulation and cell cycle control. Oncogene. 2006;25(43):5854-5863.

86. Yoshida K, Miki Y. Role of BRCA1 and BRCA2 as regulators of DNA repair, transcription, and cell cycle in response to DNA damage. Cancer Sci. 2004;95(11):866-871.

87. Thorsteinsdottir $U$, et al. Overexpression of the myeloid leukemia-associated Hoxa9 gene in bone marrow cells induces stem cell expansion. Blood. 2002;99(1):121-129.

88. Look AT. Oncogenic transcription factors in the human acute leukemias. Science. 1997; 278(5340):1059-1064.

89. Dorsam ST, et al. The transcriptome of the leukemogenic homeoprotein HOXA9 in human hematopoietic cells. Blood. 2004;103(5):1676-1684.

90. Ferrell CM, et al. Activation of stem-cell specific genes by HOXA9 and HOXA10 homeodomain proteins in CD34+ human cord blood cells. Stem Cells. 2005;23(5):644-655

91. Boudreau N, Bissell MJ. Extracellular matrix signaling: integration of form and function in normal and malignant cells. Curr Opin Cell Biol. 1998; 10(5):640-646

92. Rhoads K, Arderiu G, Charboneau A, Hansen SL, Hoffman W, Boudreau N. A role for Hox A5 in regulating angiogenesis and vascular patterning.
Lymphat Res Biol. 2005;3(4):240-252.

93. Turner NC, et al. BRCA1 dysfunction in sporadic basal-like breast cancer. Oncogene. 2007; 26(14):2126-2132.

94. Margolin S, Lindblom A. Familial breast cancer, underlying genes, and clinical implications: a review. Crit Rev Oncog. 2006;12(1-2):75-113.

95. Tan-Wong SM, French JD, Proudfoot NJ, Brown MA. Dynamic interactions between the promoter and terminator regions of the mammalian BRCA1 gene. Proc Natl Acad Sci U S A. 2008;105(13):5160-5165.

96. MacDonald G, Stramwasser M, Mueller CR. Characterization of a negative transcriptional element in the BRCA1 promoter. Breast Cancer Res. 2007;9(4):R49.

97. Bindra RS, Glazer PM. Basal repression of BRCA1 by multiple E2Fs and pocket proteins at adjacent E2F sites. Cancer Biol Ther. 2006;5(10):1400-1407.

98. Venkitaraman AR. Cancer susceptibility and the functions of BRCA1 and BRCA2. Cell. 2002; 108(2):171-182.

99. Hoshino A, et al. Effects of BRCA1 transgene expression on murine mammary gland development and mutagen-induced mammary neoplasia. Int J Biol Sci. 2007;3(5):281-291.

100.Russell AJ, et al. Alpha 6 beta 4 integrin regulates keratinocyte chemotaxis through differential GTPase activation and antagonism of alpha 3 beta 1 integrin. J Cell Sci. 2003;116(Pt 17):3543-3556.

101. Weaver VM, et al. Reversion of the malignant phenotype of human breast cells in three- dimensional culture and in vivo by integrin blocking antibodies.
J Cell Biol. 1997;137(1):231-245.

102. Paszek MJ, et al. Tensional homeostasis and the malignant phenotype. Cancer Cell. 2005;8(3):241-254.

103. Kinsella TM, Nolan GP. Episomal vectors rapidly and stably produce high-titer recombinant retrovirus. Hum Gene Ther. 1996;7(12):1405-1413.

104.Zahir N, et al. Autocrine laminin-5 ligates alpha6beta 4 integrin and activates RAC and NFkappaB to mediate anchorage-independent survival of mammary tumors. J Cell Biol. 2003;163(6):1397-1407.

105.Zufferey R, et al. Self-inactivating lentivirus vector for safe and efficient in vivo gene delivery. J Virol. 1998;72(12):9873-9880.

106. Weaver VM, et al. beta4 integrin-dependent formation of polarized three-dimensional architecture confers resistance to apoptosis in normal and malignant mammary epithelium. Cancer Cell. 2002; 2(3):205-216

107. Nikiforov MA, et al. TRRAP-dependent and TRRAP-independent transcriptional activation by Myc family oncoproteins. Mol Cell Biol. 2002; 22(14):5054-5063.

108.Sogaard TM, Jakobsen CG, Justesen J. A sensitive assay of translational fidelity (readthrough and termination) in eukaryotic cells. Biochemistry (Mosc). 1999;64(12):1408-1417.

109.Kroupis C, Stathopoulou A, Zygalaki E, Ferekidou L, Talieri M, Lianidou ES. Development and applications of a real-time quantitative RT-PCR method (QRT-PCR) for BRCA1 mRNA. Clin Biochem. 2005;38(1):50-57. 